\title{
Screening of beans (Phaseolus vulgaris L.) genotypes for drought tolerance
}

\author{
Jigme Thinley ${ }^{1}$ and Choki Dorji ${ }^{2}$ \\ ${ }^{1}$ B.Sc. Agriculture, College of Natural Resources, Lobesa \\ ${ }^{2}$ Assisstant lecturer, Rural Development Training Center, Ministry of Agriculture and \\ Forest, Zhemgang \\ 1jigmethinley1996@gmail.com \\ 2cdorji51@gmail.com
}

\begin{abstract}
Drought is an important factor limiting crop yield and food insecurity globally. Sixty-percent of the bean production areas are prone to drought and subsequently result in eighty-percent of yield reduction. It is emerging as a rising threat to farming communities in Bhutan. Limited studies on crop drought tolerance done in Bhutan. Six bean genotypes (Orey serbu, Orey regtang, Orey brokchilu, Yadhipa orey, Kerongree orey, Brokopali) chose to ascertain drought tolerance. The study was a factorial experiment with Completely Randomized Design (CRD) with six treatments and three replications. Genotypes were subjected to drought stress after 50\% flowering till the start of pod formation. The drought condition induced for ten days during the pod formation and the irrigation resumed till harvesting. Means of water-stressed genotypes compared to their corresponding non-stressed with an All-pairwise comparison using the Bonferroni test at the significant level $p<.05$. The growth (leaf area, relative water content, root length, shoot weight, maturity) and yield parameters (the number of pods, pod length, the number of seeds number per pod, seed weight) determined during the time of harvest. There were significant differences $(p<.05)$ in all the parameters measured under stress and non-stress conditions. Water-stress decreases plant growth and development of all the bean genotypes. With regards to drought susceptibility index (DSI), Orey serbu(1), Orey regtang(1), Yadhipa orey(1), and Kerongree orey(1) had the lowest DSI value. The lesser the susceptible indices, the greater is the tolerance level to the drought and vice versa.
\end{abstract}

Keywords: Drought susceptibility index, Drought stress, Tolerance

\section{Introduction}

Common beans (Phaseolus vulgaris L.) consume as a staple and low- cost protein source in the underdeveloped countries where protein malnutrition is prevalent [13]. [12] stated that the genus Phaseolus originated from the Mesoamerican regions comprises five domesticated 
species: Phaseolus vulgaris (common bean), Phaseolus dumosus, Phaseolus coccineus (runner beans), Phaseolus acutifolius (tepary beans), and Phaseolus lunatus (Lima bean). Dry beans are a staple food in Latin American, Eastern, and South African countries [2]. Similarly, beans are the source of dietary carbohydrates and micronutrients for more than 300 million people from Eastern Africa and Latin America [50,9]. India produced 6,75,188 metric tons [MT] of green beans, followed by Bangladesh (137495 MT) in 2017 [21Error! Reference source not found.]. As per [16], Bhutan produced 1,475 MT of beans in 2016 and 5,273 MT in 2017 [17]. Tsirang has the highest harvested area (475 acres), and Bumthang the least harvested area (3 acres) [17]. Bean production is adversely affected by both biotic and abiotic factors [23], and the major abiotic factor affecting crop production worldwide is water stress [28]. Water-stress is ranked second after pests and diseases in reducing the agricultural grains. Sixty- percent of the bean production areas are prone to the drought that drains an eighty-percent of yield [24, 41]. The $60 \%$ of beans production in the world occurs in the agricultural land prone to water deficit, lack of irrigation system, and the dry periods may result in an $80 \%$ yield reduction [41]. According to [17], the drought (4\% of total households) and the insufficient irrigation supply (27\% of total households) are emerging as a threat to agriculture in Bhutan. Beans are the most important, directly consumed food legume, and it is one of the most important cash crops after potato grown by every Bhutanese farmer. Almost every Bhutanese household consumes beans and remains as highly demanded in the market; however, the study by [49] reported that the drought gets severer in the future due to continuous anthropogenic activities. This study aimed to identify the drought-tolerant bean genotypes, which would help maintain the production and to meet the demand in the Bhutanese market, and provide the basis for the development of drought-tolerant hybrids in the future.

\section{Materials and Methods}

\subsection{Study site, experimental design, stress treatment, and irrigation schedule}

The greenhouse of the agriculture farm of College of Natural Resources (CNR), Lobesa, was used to perform this study in August 2019. It is at an elevation of 1450 meters above sea level between $27^{\circ} 30^{\prime} 1^{\prime \prime} \mathrm{N}$ and $89^{\circ} 52^{\prime} 42^{\prime}$ ' E of Greenwich. The area experiences an annual rainfall of $500 \mathrm{~mm}$ to $1500 \mathrm{~mm}$ with temperatures ranging from $5^{\circ} \mathrm{C}$ to $30^{\circ} \mathrm{C}$. It lies in the dry subtropical region and experiences hot and humid summers during the monsoon months of June, July, and August. The bean varieties used for the experiment were Yadhipa orey(13), Orey 
serbu(27), Kerongree orey(7), Orey regtang(6), Orey brokchilu(15), and Bropali(25). Used completely randomized design (CRD) for this factorial experiment. There were six treatments (six bean genotypes) and three replications (three plants per replica) and classed treatments into the water-stressed and nonstressed groups. Used a total of 108 potted plants during the experiment, and the distance between the pots was $30 \mathrm{~cm}$ within rows and $40 \mathrm{~cm}$ between rows [4]. For media, used $3 \mathrm{~kg}$ of topsoil to fill 4,416 cm3 volume plastic pots. Subjected genotypes to drought stress after 50\% flowering and lasted for ten days, and resumed irrigation till harvesting similar to [4, 8]. Weighed pots following one-day intervals [15] and applied the water by calculating the required amount of water using the formula; the amount of water = the weight of pot at field capacity - pot weight at field condition and maintained the soil moisture near field capacity (FC) throughout the experiment [8].

\subsection{Data collection and analyses}

Recorded the weather data using Datalogger (temperature and relative humidity) and recorded both growth and yield attribute data during physiological maturity that defines the number of days for $75 \%$ to $90 \%$ of pods to lose their green pigmentation [48]. Collected data on leaf area, the number of pod per plant, weight of seed per plant, shoot dry weight, root dry weight, and calculated drought susceptibility index (DSI).

Calculated drought Susceptibility index (DSI) using the following formula:

$$
\begin{aligned}
\mathrm{DSI} & =1-\mathrm{Y}_{\mathrm{d}} / \mathrm{Y}_{\mathrm{w}} \div \mathrm{D} \\
\mathrm{D} & =1-\frac{\text { Mean yield under drought stress }(\text { Grand mean })}{\text { Mean yield under well watered }(\text { Grand mean })}
\end{aligned}
$$

Where

$\mathrm{D}=$ drought intensity

$\mathrm{Y}_{\mathrm{d}}=$ Mean yield under drought condition (Average yield of genotypes

$\mathrm{Y}_{\mathrm{w}}=$ Mean yield under well-watered condition. (Average yield of each genotype)

Data were subjected to a two-way analysis of variance (Two-way ANOVA) to compare differences in the means. Calculated statistical significances at $\mathrm{p}<.05$ and collated group means following Bonferroni pairwise comparison tests. The factorial design in statistical program Statistix version 8 used for all the analyses.

\section{Results and Discussion}

\subsection{Effect of drought stress on leaf area $\left(\mathrm{dm}^{2}\right)$}


The significant difference in leaf area was recorded between the treatments group (stress and non stress) $[F(1,22)=52.96, p<.05]$ and within the genotypes group $[F(5,22)=10.42, P<$ .05]. There was a significant interaction between the treatment and genotypes $(p<.05)$. Results indicate the genotype difference in leaf area was affected by the drought stress. Under the waterstressed condition, most of the genotypes had a smaller leaf area than non-stress [Figure 1]. Orey serbu $\left(M=0.55 \mathrm{dm}^{2}, S D \pm 0.05\right)$ and Orey regtang $\left(M=0.55 \mathrm{dm}^{2}, S D \pm 0.03\right)$ recorded significantly higher in leaf area under water stress. However, there was no significant difference $(p>.05)$ between genotypes Yadhipa orey, Orey brokchilu, and brokpali in stress conditions. Wilting, shedding, and curling of leaves, closure of stomata, and reduction of cell enlargement could be the reason for the reduction in leaf area under the stress condition. During the onset of water stress, it inhibits cell elongation in the leaf, and the lower leaf area leads to less water uptake from the soil, and transpiration is reduced [20]. Under the non-water stressed condition genotypes, Orey serbu had recorded significantly higher in leaf area $\left(M=0.75 \mathrm{dm}^{2}, S D \pm 0.01\right)$. The result coincided with [42, 15], who observed that plants grown under water deficit conditions showed a lower leaf area than plants grown under control conditions. [43] argued that the leaf area is related to plant metabolism, dry matter production, and yield.

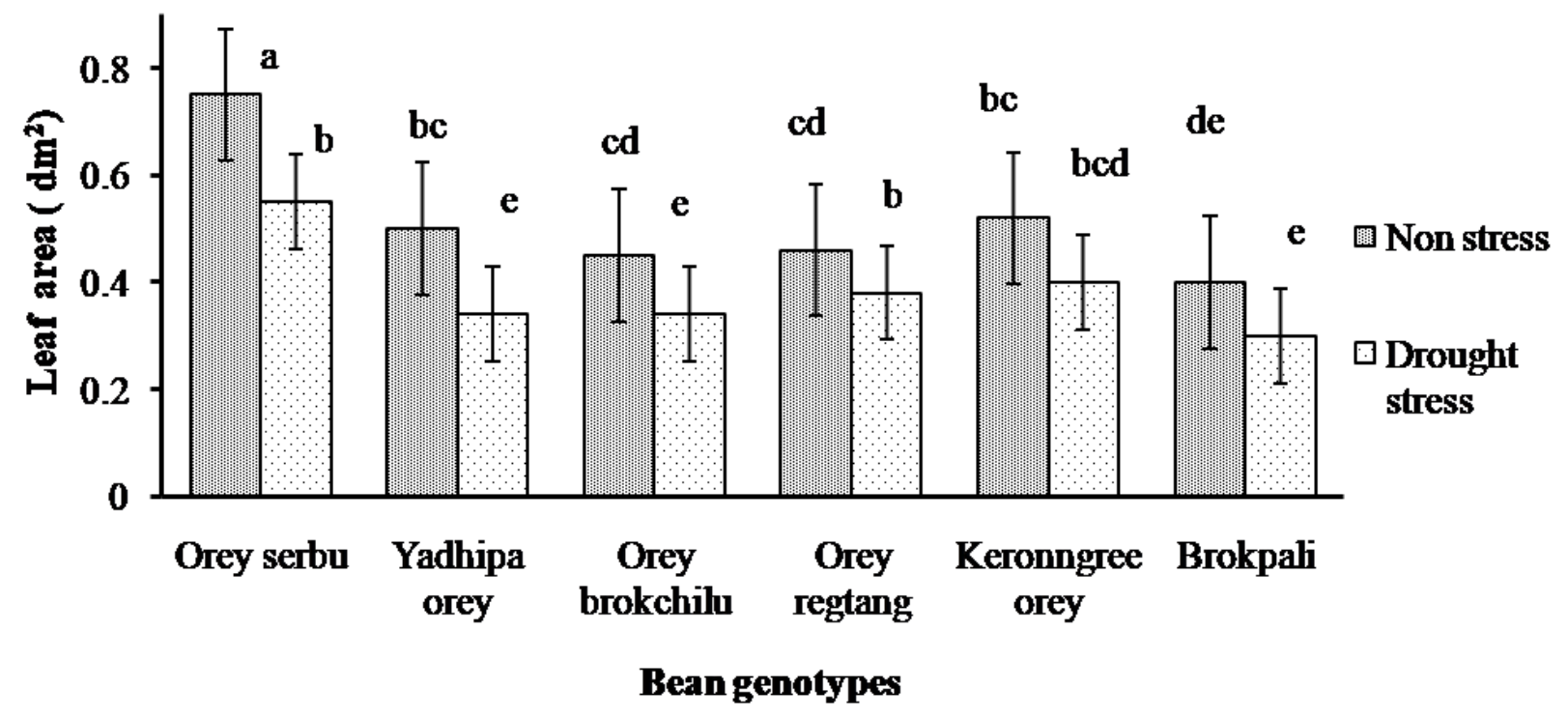

Figure 1: Leaf Area ( $\left.\mathbf{d m}^{2}\right)$.

\subsection{Effect of drought stress on pod number}

There was a significant difference in the number of pods per plant in both between treatments and genotypes group $[F(1,22)=64.61, P<.05][F(5,22)=8.67, p<.05]$. The study revealed 
the significant $(p<.05)$ interaction between treatment and genotypes and shows that the varietal difference in pod number was affected by stress imposed [Figure 2]. Under the water-stressed condition, the number of pods per plant decreased compared to non-stress. The highest average number of pods was in Yadhipa orey $(M=5.25, S D \pm 0.33)$. The fewer floral part formation could have affected the number of pods per plant. Under the non-water stressed condition, Orey regtang had the highest $(M=7.39, S D \pm 0.98)$ average number of pods per plant. The present findings are in line with [13], who reported that under the high moisture stress during the reproductive stage, the plants are exposed to floral abortion resulting in low yield. Other authors $[7,44]$ also reported that water-stress imposed during the flowering and pod setting causes flower and pod abortion. The result agrees with the finding of [7, 42, 5] and [29] reported the decline in pod number when irrigated in 21 days intervals. According to [33], the reproductive stage is the most sensitive to drought stress.

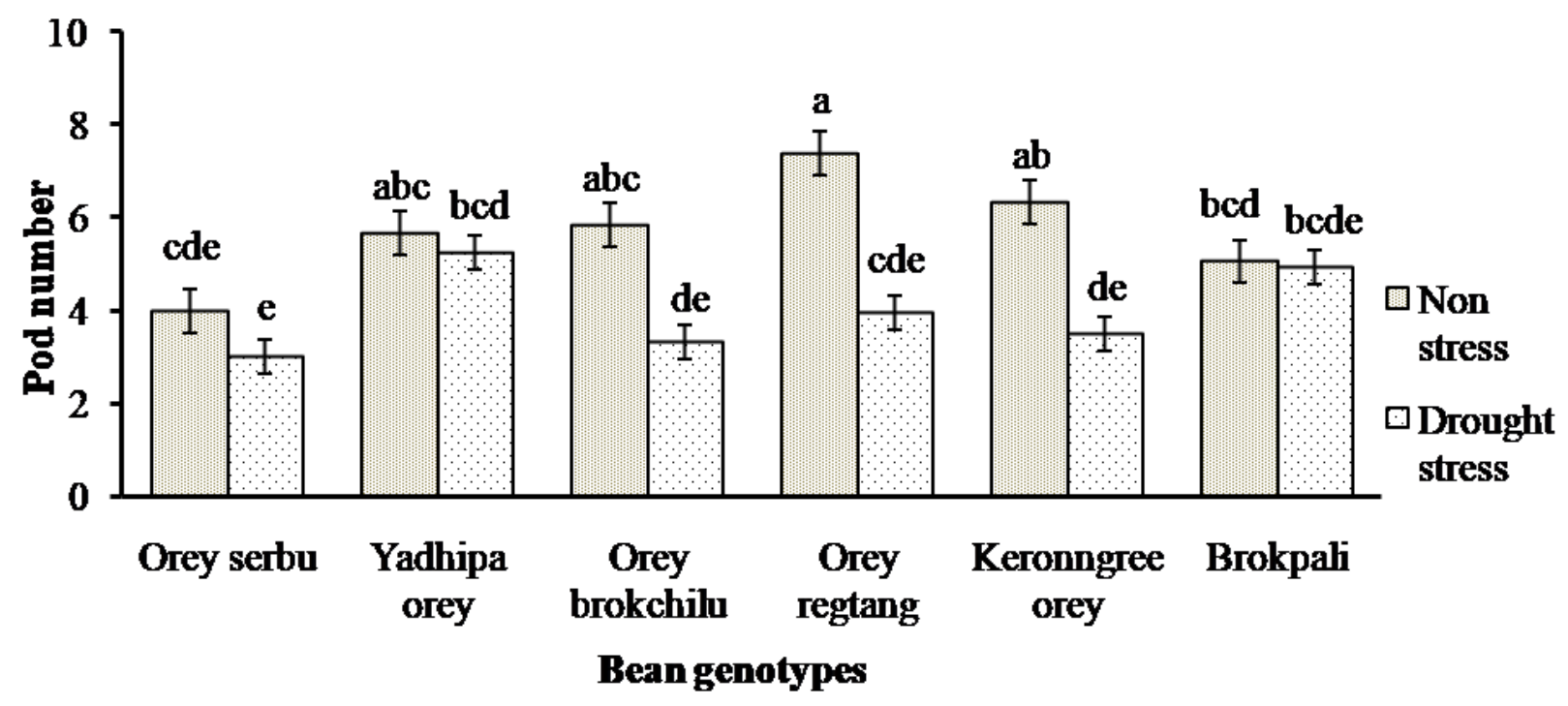

Figure 2: Number of pods per plant.

\subsection{Effect of drought stress on seed weight $(g)$}

There was a highly significant difference in seed weight both between treatment and within genotypes group $[F(1,22)=597.64, p<.05][F(5,22)=35.76, p<.05]$. Under the stress condition, seed weight/yield decrease compared to non-stress [Figure 3]. However, Brokpali recorded the highest seed weight $(M=5.94 \mathrm{~g}, S D \pm 0.59)$. The reduction in seed weight/yield under the water stress was may be due to a decrease in carbohydrate assimilation that might have lead to less pod number. Under the non-water stress condition, Brokpali had the highest average 
seed weight of $(M=14.51 \mathrm{~g}, S D \pm 1.51)$. The reduction in a legumes seed yield under the stress condition was due to the lower number of pods per plant [27, 38, 30, 7]. The reduction in the seed yield and the number of pods is due to the detrimental effects of drought on pod set and grain filling [14].

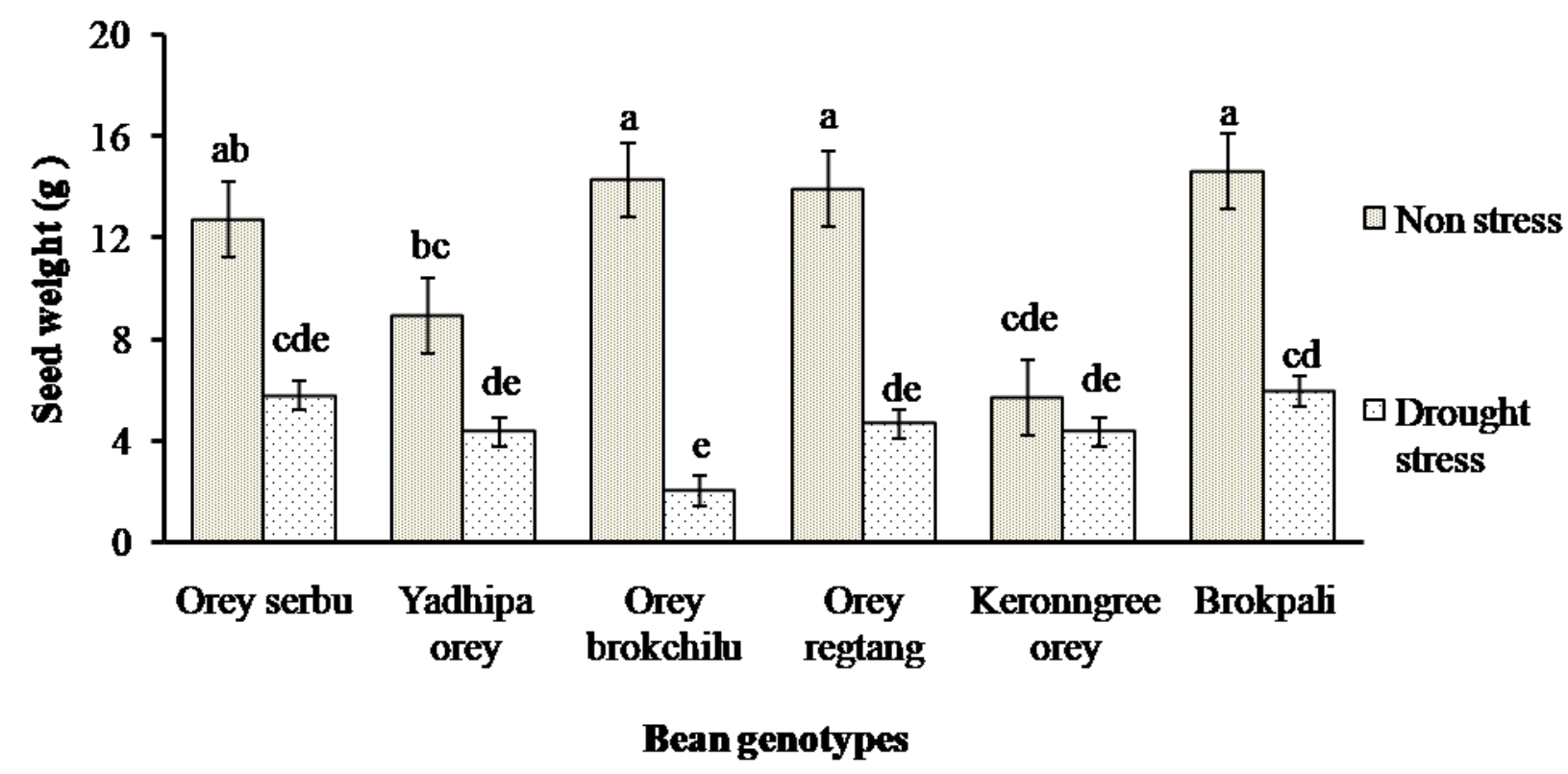

\section{Figure 3: Seed weight (g) per plant.}

A similar result obtained by [46], yield reduction in common beans of $58-87 \%$ occurs when the water stress is set forth during its reproductive stage. The result accords with the findings of [32, 45, 3, 1]. [24] also had a similar observation in physiological responses of common bean (Phaseolus vulgaris 1.) genotypes to water stress. Moreover, reported seed yield reduction under water stress for a black gram and green gram [47], soybean [26], and Phaseolus vulgaris [36, 22, 15]. The lower seed yield in common beans under stress is due to the lower photosynthate assimilation and carbohydrate partitioning to the developing grain due to drought stress [37, 40, $6]$.

\subsection{Effect of drought stress on dry weight (g) of the shoot}

The study revealed a significant difference in shoot weight between the treatment group $[F(1$, $22)=40.15, p<.05]$ and the genotypes group $[F(5,22)=13.81, p<.05]$. Under the water stress condition, plant shoot dry weight decreased significantly than non-stress [Figure 4]. It could be due to limited photosynthate assimilation and the limited amount of water absorbed by roots. Orey serbu was observed the highest shoot weight of $(M=6.91 \mathrm{~g}, S D \pm 0.14)$ under water stress. 
For non-water stress conditions, Brokpali had the highest shoot weight ( $M=14.61 \mathrm{~g}, S D \pm 0.29)$. A similar finding was investigated by $[35,10]$ there is more reduction in shoot growth than root growth under stress. According to [46], both shoots and roots are the most affected parts of plants and key components of the adaptation to drought at the morphological level.

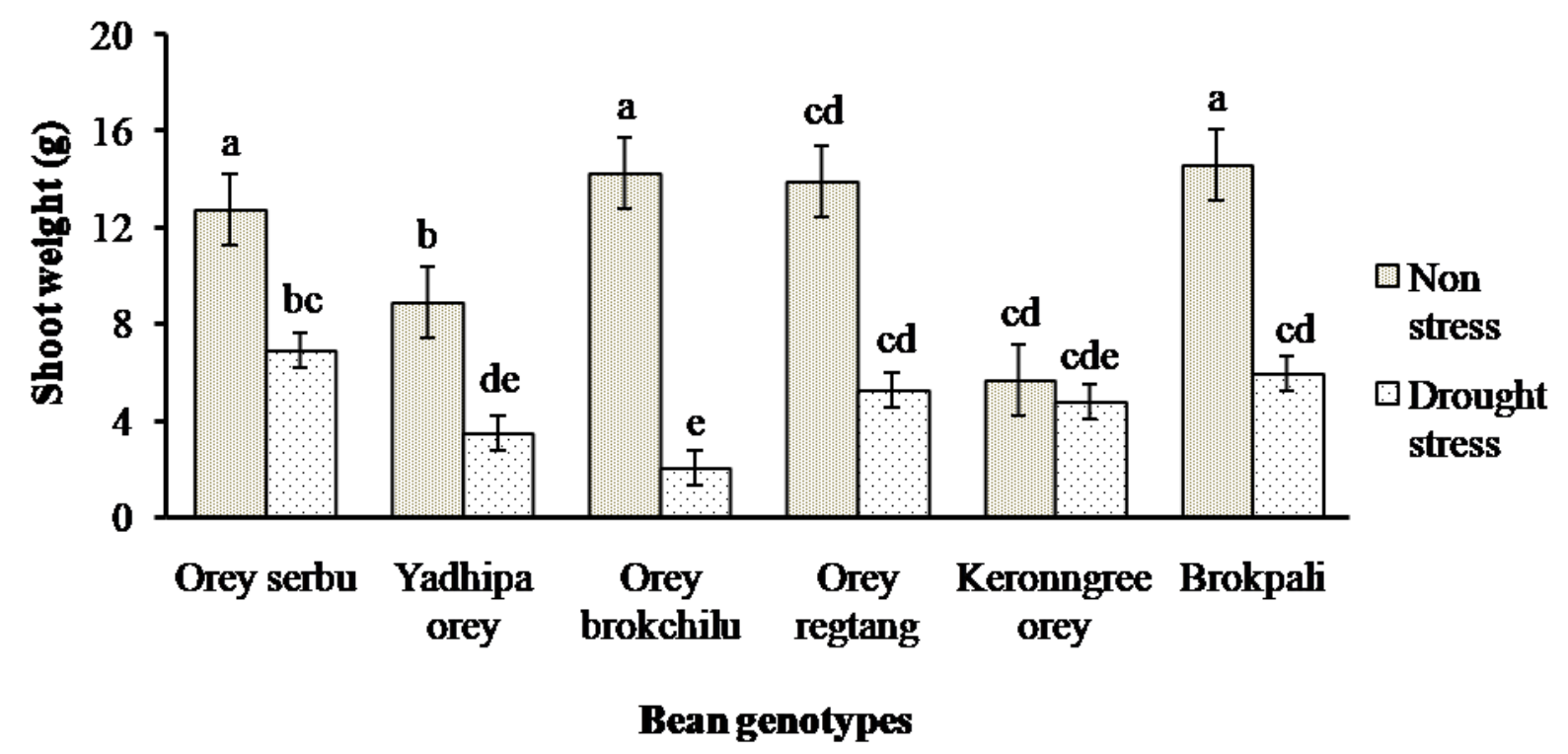

\section{Figure 4: Shoot weight (g)}

Drought stress severely reduces stem growth and the ability to intercept solar radiation [1]. Among the genotypes, in stress conditions, Orey serbu, Brokpali, and orey regtang showed significantly higher shoot dry weight than the other genotypes. According to [11], a positive correlation $\left(\mathrm{r}=0.53^{* *}\right)$ exists between shoot and root dry weight in optimal irrigation. However, no correlation $(\mathrm{r}=0.11)$ exists under drought stress. It could be due to the genotypic difference in terms of root adjustment to stress.

\subsection{Effect of drought stress on root dry weight $(g)$}

Significant difference $(p<.05)$ was observed in the root weight both between the treatment and genotypes group $[F(1,22)=135.47, p<.05][F(5,22)=6.36, p<.05]$. Under water-stressed conditions, root dry weight decreased compared to non-stress [Figure 5]; however, Brokpali had the highest root weight $(M=1.84 \mathrm{~g}, S D \pm 0.36)$ under water stress. The decreased in root dry weight could be due to poor roots proliferation in a stressful environment. Plants grown under water deficit conditions had low root biomass [34]. For non-water stress conditions, Keronngree orey recorded the highest root weight $(M=2.9 \mathrm{~g}, S D \pm 0.23)$. 


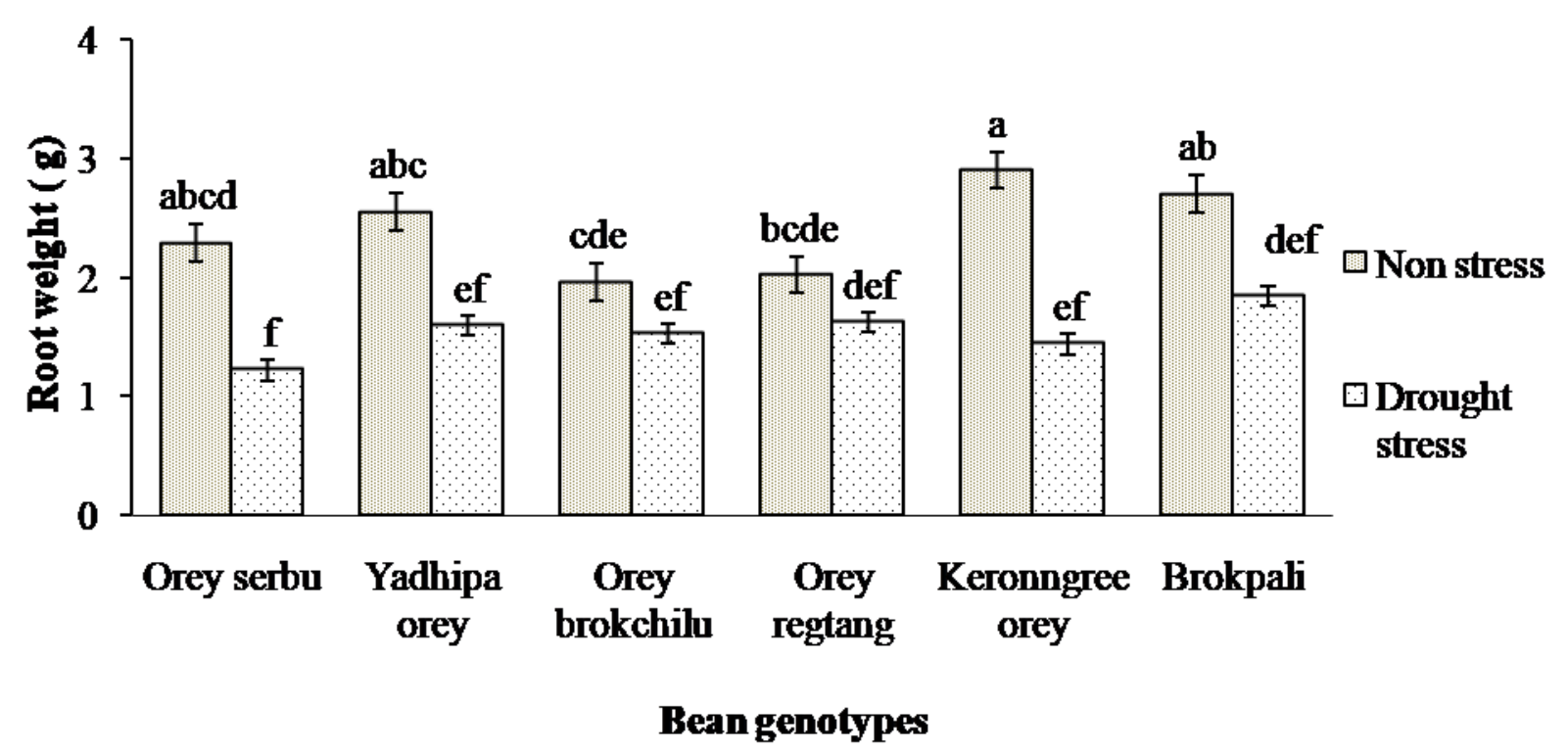

Figure 5: Root weight (g).

Yield components and Biomass accumulation in beans are affected by drought stress [31]; however, few genotypes under the stress condition seem to perform better (Brokpali (1.84 g), Orey regtang (1.62 g), and Yadhipa orey $(1.60 \mathrm{~g}))$. It could mean that these genotypes have better soil water acquiring efficiency compared to other genotypes. According to [18], the number of roots decreases with a decrease in water potential. Moreover, reduction in lateral roots directly affects the root biomass [39]. The root characters (biomass, length, density, and depth) are the main drought avoidance traits that contribute to the final yield under terminal drought environments [25].

3.6 Drought intensity (D) and Drought susceptibility Index (DSI)

Determined the drought susceptibility index (DSI) on relative water content, shoot weight, seed weight, root weight, leaf area, pod number, pod length, seed number, and root length for six water-stressed genotypes. The Drought susceptibility index (DSI) for [Table 1] leaf area ranged from -0.27 to $0.27,-0.13$ to 1.45 for shoot weight, 0.56 to 1.43 for root weight, 0.05 to 2.14 for relative water content, 0.38 to 1.41 for seed weight, -0.41 to 0.22 for pod number, -0.06 to 0.05 for pod length, -0.23 to 0.2 for seed number, and -0.32 to 0.17 for root length. The smaller the value of the drought susceptibility index, the more tolerant is the genotypes to water stress conditions. 
Table 1: Drought susceptibility index (DSI)

\section{DSI}

Genotypes

\begin{tabular}{rrrlrrrrr}
\hline LA & SHW & RW & RWC & SW & PN & PL & SN & RL \\
\hline-0.18 & -0.13 & 1.33 & 0.11 & 0.89 & -0.09 & -0.01 & 0.13 & 0.14
\end{tabular}

Yadhipa orey

$-0.27 \quad 1.03 \quad 1.06$

2.14

0.84

$-0.34-0.04$

$-0.18 \quad 0.17$

Orey brokchilu

$-0.15$

1.45

0.62

1.57

1.41

0.17

0.05

$0.17 \quad 0.04$

Orey regtang

0.27

1.05

0.56

0.05

1.09

$0.22 \quad-0.02$

$\begin{array}{ll}-0.23 & -0.32\end{array}$

Kerongree orey

0.058

0.27

1.43

1.18

0.38

$0.19 \quad-0.06$

$-0.17 \quad-0.15$

Brokpali

0.034

1.01

0.91

0.63

$\begin{array}{lll}0.97 & -0.41 & 0.03\end{array}$

$0.2 \quad-0.05$

LA: Leaf area, SHW: Shoot weight, RW: Root weight, RWC: Relative water content, and SW:

Seed weight

\subsection{Scoring and ranking of genotypes based on drought susceptibility indices}

Ranked the water-stress toleration of six bean genotypes [Table 2]; the scoring and ranking were such that the least susceptible genotypes scored 1, and the highest susceptible genotypes score 6 [Table 4]. Values of Drought Susceptibility Index (DSI) less than 1.0 indicate toleration to drought. Values of DSI equal to 0.0 indicate maximum possible drought tolerance (no effect of drought on yield). Four genotypes (Orey serbu, Yadhipa orey, Orey regtang, and Kerongree orey) had the least DSI (rank 1st) and have a greater drought tolerance level. Orey brokchilu had the highest DSI (ranked 6th), followed by Brokpali (ranked 5th) and more susceptible to drought conditions.

Table 2: Scoring and ranking of genotypes

Rank Ran

$\begin{array}{llllllllllll}\text { Genotypes } & \text { LA } & \text { SHW } & \text { RW } & \text { RWC } & \text { SW } & \text { PN } & \text { PL } & \text { SN } & \text { RL } & \text { sum } & k\end{array}$

$\begin{array}{llllllllllll}\text { Orey serbu } & 2 & 1 & 5 & 2 & 3 & 3 & 4 & 4 & 5 & 29 & 1^{\text {st }}\end{array}$

$\begin{array}{lllllllllllll}\text { Yadhipa orey } & 1 & 4 & 4 & 6 & 2 & 2 & 2 & 2 & 6 & 29 & 1^{\text {st }}\end{array}$

$\begin{array}{lllllllllll}\text { Orey brokchilu } & 3 & 6 & 2 & 5 & 6 & 4 & 6 & 5 & 4 & 41\end{array} 6^{\text {th }}$

$\begin{array}{lllllllllllll}\text { Orey regtang } & 6 & 5 & 1 & 1 & 5 & 6 & 3 & 1 & 1 & 29 & 1^{\text {st }}\end{array}$

Kerongree

\begin{tabular}{|c|c|c|c|c|c|c|c|c|c|c|c|}
\hline orey & 5 & 2 & 6 & 4 & 1 & 5 & 1 & 3 & 2 & 29 & $1^{\text {st }}$ \\
\hline Brokpali & 4 & 3 & 3 & 3 & 4 & 1 & 5 & 6 & 3 & 32 & $5^{\text {th }}$ \\
\hline
\end{tabular}


LA: Leaf area, SHW: Shoot weight, RW: Root weight, RWC: Relative water content, and SW: Seed weight

\section{Conclusion}

The present study revealed Orey serbu (rank 1st), Yadhipa orey (rank 1st), Orey regtang (rank 1st), and Kerongree orey (rank 1st) would perform better under conditions of limited water supply based on their low drought susceptibility index in the greenhouse. Brokpali (rank 5th) and Orey brokchilu (rank 6th) were least tolerant to drought. Leaf area, the number of pods, the number of seeds, and seed weight per plant was significantly reduced by drought stress compared to non-stress. Drought stress affected the proper growth and development and ultimately affected the yield of the plants.

\section{Acknowledgment}

We would like to extend our heartfelt gratitude to the Department of Agriculture, College of Natural Resources, and lab assistant for their supervision, suggestion, advice, guidance, encouragement, and commitment during the research work. Lastly, we acknowledge lecturers, friends, and my family for supporting our study.

\section{References}

1. Abrokwah AO. Screening of maize (Zea mays L.) inbreds lines for tolerance to drought [dissertation]. Ghana (GH): Kwame nkrumah university of science and technology; 2016 [cited 2020 Apr 15]. Available from: http://ir.knust.edu.gh/handle/123456789/7557

2. Aggarwal VD, Pastor-Corrales MA, Chirwa RM, Buruchara RA. Andean beans (Phaseolus vulgaris L.) with resistance to angular leaf spot pathogen (Phaeoisariopsis griseola) in southern and eastern Africa. Euphytica 2004; 136: 201-210. doi: 10.1023/B:EUPH.0000030671.03694.bb

3. Ahmad A, Selim M, Aldrfasi A, Afzal M. Effect of drought stress on mungbean (Vigna radiata L.) under arid climatic conditions of Saudi Arabia. Ecosystems and Sustainable Development 2015; 192: 185-193. doi: 10.2495/ECO150171

4. Alidu MS. Evaluation of Cowpea genotypes for drought tolerance using the pot screening approach. Asian Research Journal of Agriculture 2018; 10(2): 1-11. doi: $\underline{10.9734 / \mathrm{ARJA} / 2018 / 45806}$ 
5. Ardakani LG, Farajee H, Kelidari A. The effect of water stress on grain yield and protein of spotted bean (Phaseolus vulgaris L.), cultivar Talash. International Journal of Advanced Biological and Biomedical Research 2013; 1(9): 940-949.

6. Asfaw MM, Blair MW, Struick P. Multi-environment quantitative traits locus analyses for photosynthate acquisition, accumulation and remobilization traits in a common beans, Genes Genomes Genetic 2012; 1(2): 579-595. doi: 10.1534/g3.112.002303

7. Barrios AN, Hoogenboom G, Nesmith DS. Drought stress and the distribution of vegetative and reproductive traits of a bean cultivar. Sci. Agric. (Piracicaba, Braz.)2005; 62(1). doi: $\underline{10.1590 / \mathrm{S} 0103-90162005000100004}$

8. Batieno BJ, Tignegre JB, Hamdou S, Hamadou Z, Ouedraogo TJ, Danquah E, Ofori K. Field Assessment of Cowpea Genotypes for Drought Tolerance. International Journal of Sciences: Basic and Applied Research (IJSBAR) 2016; 30(4): 358-369.

9. Beebe SE, Rao IM, Blair MW, Acosta-Gallegos JA. (2013). Phenotyping common beans for adaptation to drought. Frontiers in Physiology 2013, 4; [about 35 p]. doi: $\underline{10.3389 / \text { fphys.2013.00035 }}$

10. Bibi A, Sadaqat, H.A., Akram, H.M. and Maarout, A. Physiological markers for screening sorghum (Sorghum bicolor) germplasm under water stress condition. Int J Agric Biol 2010; 12: 451-455. Available from: https://www.researchgate.net/publication/228423665_Physiological_Markers_for_Screen ing_Sorghum_Sorghum bicolor_Germplasm_under_Water_Stress_Condition

11. Brdar-Jokanović M, Girek Z, Pavlović S, Ugrinović M, Zdravković J. (Shoot and root dry weight in drought exposed tomato populations. Genetika 2014; 46(2): 495-504. doi: 10.2298/GENSR1402495B

12. Broughton WJ, Hernandez G, Blair M, Beebe S, Gepts P, Vanderleyden J. Beans (Phaseolus spp.) - model food legumes. Plant and Soil 2003; 252 (1): 55-128 doi: 10.1023/A:1024146710611

13. Castaneda-Saucedo MC, Cordova-Tellez L, Gonzalez-Hernandez VA, Delga-Alvardo A, Santacruz-Varela A, Santos GG. (2009). Physiological performance, yield.and quality of dry bean seeds under drought stress. Interciencia 2009; 34(10): 748-754. 
14. Chiulele RM. Breeding cowpea (Vigna unguiculata (L.) walp.) for improved drought tolerance in Mozambique [Ph.D. thesis]. KwaZulu-Natal (KZN): University of KwaZuluNatal; 2010.

15. Choudhury AK, Karim MA, Moynul M, Haque QA, Khalio QA, Ahmed JU, Hossain MM. Effect of water stress on plant water status of french bean (phaseolus vulgaris 1.). Indian journal of plant physiology 2010; 15(2): 131-136.

16. Department of Agriculture (DoA). Agriculture Statistic 2016. Thimphu, Bhutan: DoA, Ministry of Agriculture and Forestry; 2016.

17. Department of Agriculture (DoA). Agriculture Statistic 2017. Thimphu, Bhutan: DoA, Ministry of Agriculture and Forestry; 2017.

18. Dhole VJ, Reddy KS. Gamma rays induced moisture stress tolerant long root mutant in mungbean (Vigna radiata L Wilczek). Electronic Journal of Plant Breeding 2010; 1(5):1299-1305.

19. Drewnowski A. The cost of US foods as related to their nutritive value. The American Journal of Clinical Nutrition 2010; 92(5): 1181-1188. doi: 10.3945/ajen.2010.29300.

20. Fathi A, Tari DB. Effect of Drought Stress and its Mechanism in Plants. International Journal of life science 2016; 10(1). 1-6. doi: 10.3126/ijls.v10i1.14509

21. Food and Agriculture Organization of the United Nations [FAO] (2017). FAOSTAT, 2017 [cited 2020 Mar 27]. Available from https://www.fao.org/faostat/en/\#compare

22. Islam MS, Haque MM, Khan MM, Hidaka T, Karim MA. Effect of fertilizer potassium on growth, yield and water relations of French bean (Phaseolus vulgaris L.) under water stress conditions. Japanese Journal Tropical Agriculture 2004; 48: 1-9. Japanese. doi: $\underline{10.11248 / \text { jsta1957.48.1 }}$

23. Jaleel CA, Manivannan P, Wahid A, Farooq M, Somasundaram R, Panneerselvam R. Drought stress in plants: a review on morphological characteristics and pigments composition. Int J Agric Biol 2009; 11:100-105.

24. Kalima SP. Physiological responses of common bean (Phaseolus vulgaris L.) genotypes to water stress [MSc Thesis]. Lusaka, Zambia: University of Zambia; 2013.

25. Kavar T, Maras M, Kidric M, Sustar-Vozlic J, Meglic V. Identification of genes involved in the response of leaves of Phaseolus vulgaristo drought stress, Mol. Breeding 2008; 21: 159-172. doi: 10.1007/s11032-007-9116-8 
26. Liu F, Andersen MN, Jensen CR. Loss of pod set caused by drought stress is associated with water status and ABA content of reproductive structures in soybean. Functional Plant Biology 2003; 30(3): 271-280. Available from: https://www.publish.csiro.au/fp/FP02185

27. Lopez FB, Johansen C, Chauhan YS. (1996). Effect of timing of drought stress on phenology, yield and yield components of a short-duration pigeon pea. Journal of Agronomy \& Crop Science 1996; 177(5): 311-320. doi: 10.1111/j.1439037X.1996.tb00251.x

28. Man D, Bao Y, Han. Drought tolerance associated with proline and hormone metabolism in two Fescue cultivar. American Society for Horticulture Science 2011; 46(7):10271032). doi: $10.21273 /$ HORTSCI.46.7.1027

29. Martínz JP, Silva H, ledent JF, Pinto M. Effect of drought stress on the osmotic adjustment, cell wall elasticity and cell volume of six cultivars of common beans (Phaseolus vulgaris L.). Europ. J. Agronomy 2007; 26(1): 30-38. doi: 10.1016/j.eja.2006.08.003

30. Muchow RC. Phenology, seed yield and water use of grain legumes grown under different water regimes in a semiarid tropical environment. Field Crops Res. 1985; 11: 81-97. doi: 10.1016/0378-4290(85)90093-0

31. Munoz-Perea CG, Tera'n H, Allen RG, Wright JL, Westermann DT, Singh SP. Selection for drought resistance in dry bean landraces and cultivars. Crop Science 2006; 46: 2111 2120. doi: 10.2135/cropsci2006.01.0029

32. Nam NH, Chauhan YS, Johansen C. Effect of timing of drought stress on growth and grain yield of extra-short-duration pigeonpea lines, J. Agr. Sci. 2001; 136: 179-189.

33. Nielsen DC, Nelson. Black bean sensitivity to water stress at various growth stages. Crop $\quad$ Science $1998 ; \quad 38(2): \quad 422-427 . \quad$ doi: 10.2135/cropsci1998.0011183X003800020025x

34. Nleya TM, Slinkard AE, Vandenberg A. Differential performance of pinto bean under varying levels of soil moisture. Canadian Journal of Plant Science 2001; 81(2): 233-239. doi: $\underline{10.4141 / \mathrm{P} 99-180}$ 
35. Okçu G, Kaya MD, M. Atak, M. (2005). Effects of salt and drought stresses on germination and seedling growth of pea (Pisum sativum L.). Turk J. Agric. For. 29: 237242.

36. Omae H, Kumar A, Egawa Y, Kashiwaba K, Shono M. Midday drop of leaf water content to drought tolerance in snap bean (Phaseolus vulgaris L). Plant Prod. Sci. 2005; 8(4): 465-467. Doi: 10.1626/pps.8.465

37. Perea-Munoz GC, Allen GR, Westermann TD, Wright SP, Singh. Water use efficiency among drybean land races and cuktivars in drought stressed and non stressed environment. Euphytica 2007; 155: 393-402. doi: 10.1007/s10681-006-9340-Z

38. Pilbeam CJ, Akatse JK, Hebblethwaite PD, Wright CD. Yield production in two contrasting forms of spring-sown faba beans in relation to water supply. Field Crops Research 1992; 29(4): 273-287. doi: 10.1016/0378-4290(92)90030-D

39. Ranawake AL, Amarasingha UGS, Rodrigo WDRJ, Rodrigo UTD, Dahanayaka N.mEffect of water stress on growth and yield of mung bean (vigna radiate 1). Tropical agriculture research and extension 2001; 14(4) [about 4 p].

40. Rezene Y, Gebeyehu S, Zelleke H. Genetic variation for drought resistance in a small red seeded common bean genotypes. Africa crop science journal 2011; 19(4): 3030-312.

41. Rosales MA, Ocampo E, Rodriguez-Valentin R, OlveraCarrillo Y, Acosta-Gallegos J, Covarrubias AA. Physiological analysis of common bean (Phaseolus vulgaris L.) cultivars uncovers characteristics related to terminal drought resistance. Plant Physiol Biochem. 2012; 56: 24-34. doi: 10.1016/j.plaphy.2012.04.007

42. Samwel J. Identification of drought torelant varieties of common bean (phaseolus vulgaris 1.) in Tanzania [dissertation]. Morogoro, Tanzania: Sokoine University of agriculture; 2008.

43. Severino SL, Cardoso DG, Vale DSL, Santos DWJ. Method of determining leaf area of castor bean. Revista Brasileira de Oleaginosas e Fibrosas 2004; 8(1): 753-762.

44. Singh SP. Selection for water stress in interracial populations of common bean. Crop Science $1995 ; 38:$ 118-124.

45. Soureshjani HK, Nezami A, Kafi M, Tadayon M. Responses of two common bean(Phaseolus vulgaris L.) genotypes to deficit irrigation. Agricultural Water Management 2019; 213: 270-279. doi: 10.1016/j.agwat.2018.09.038 
46. Tekle TA, Alemu AM. Drought Tolerance Mechanisms in Field Crops. World Journal of Biology and Medical Sciences 2016; 3(2), 15-39.

47. Tripurari P, Yadav DS. Effect of irrigation and planting density on yield attributes and yield of green gram and black gram. Indian Journal of Agronomy 1990; 35: 99-101.

48. Vallejo PR, Kelly JD. Traits related to drought resistance in common beans. Euphytica 1998; 99: 127-136. doi: 10.1023/A:1018353200015

49. Wanders N, Wada Y. Human and climate impacts on the $21^{\text {st }}$ century hydrological drought. Journal of Hydrology 2015; 526: 208-220. doi: 10.1016/j.jhydrol.2014.10.047

50. Welch RM, House WA, Beebe S, Cheng Z. Genetic selection for enhanced bioavailable levels of iron in bean (Phaseolus vulgaris L.) seeds. J. Agric. Food Chem. 2000; 48(8): 3576-3580. doi: $10.1021 / \mathrm{jf0000981}$

Supporting Documents

Table 3: Data of Control

\begin{tabular}{|c|c|c|c|c|c|c|c|c|c|c|c|c|}
\hline $\begin{array}{l}\text { V } \\
\text { ar } \\
\text { ie } \\
\text { ty }\end{array}$ & $\begin{array}{l}\text { Re } \\
\text { pli } \\
\text { cat } \\
\text { ion }\end{array}$ & $\begin{array}{l}\text { No } \\
\text {.of } \\
\text { pla } \\
\text { nts }\end{array}$ & $\begin{array}{l}\text { Pod } \\
\text { lengt } \\
\text { h } \\
(\mathrm{cm}) \\
(\mathrm{PL})\end{array}$ & $\begin{array}{l}\text { No.of } \\
\text { pods/ } \\
\text { plant } \\
\text { ( PN) }\end{array}$ & $\begin{array}{l}\text { No. } \\
\text { of } \\
\text { seeds/ } \\
\text { pod } \\
(\mathrm{SN})\end{array}$ & $\begin{array}{l}\text { Seed } \\
\text { weight/ } \\
\text { plant(g } \\
)(\mathrm{SW})\end{array}$ & $\begin{array}{l}\text { Leaf } \\
\text { area } \\
(\mathrm{dm} 2) \\
(\mathrm{LA})\end{array}$ & $\begin{array}{l}\mathbf{R} \\
\mathbf{W} \\
\mathbf{C}( \\
\% \\
)\end{array}$ & $\begin{array}{l}\text { Root } \\
\text { lengt } \\
\text { h } \\
\text { (cm) } \\
\text { (RL) }\end{array}$ & $\begin{array}{l}\text { Root } \\
\text { weigh } \\
\text { t(g) } \\
(\text { RW) }\end{array}$ & $\begin{array}{l}\text { Shoot } \\
\text { weigh } \\
\text { t } \\
\text { (g)(S } \\
\text { HW) }\end{array}$ & $\begin{array}{l}\text { mat } \\
\text { urit } \\
\text { y } \\
\text { (Da } \\
\text { ys) }\end{array}$ \\
\hline 1 & 1 & 1 & 12 & 4 & 3.5 & 3 & 0.43 & $\begin{array}{r}53 . \\
1\end{array}$ & 25 & 2.46 & 2.17 & 50 \\
\hline 1 & 1 & 2 & 13.6 & 3 & 4.6 & 3 & 0.32 & $\begin{array}{r}39 . \\
4\end{array}$ & 26 & 2.4 & 3.95 & 49 \\
\hline 1 & 1 & 3 & 12.2 & 3.5 & 4.6 & 3 & 0.37 & $\begin{array}{r}45 . \\
6\end{array}$ & 26 & 2.43 & 1.95 & 51 \\
\hline $\begin{array}{l}\text { A } \\
\text { ve } \\
\text { ra } \\
\text { ge }\end{array}$ & & & 12.6 & 3.5 & 4.2 & 3.0 & 0.37 & $\begin{array}{r}46 . \\
03\end{array}$ & 25.7 & 2.43 & 2.69 & 50 \\
\hline 1 & 2 & 1 & 13.5 & 4 & 2.5 & 11 & 0.73 & 37 & 30 & 1.3 & 2.52 & 58 \\
\hline 1 & 2 & 2 & 13.2 & 4 & 3.5 & 12 & 0.85 & $\begin{array}{r}36 . \\
85\end{array}$ & 30 & 1.84 & 1.75 & 56 \\
\hline 1 & 2 & 3 & 12.2 & 4 & 3.5 & 10 & 0.79 & $\begin{array}{r}36 . \\
7\end{array}$ & 30 & 1.99 & 1.87 & 57 \\
\hline $\begin{array}{l}\text { A } \\
\text { ve } \\
\text { ra }\end{array}$ & & & 12.97 & 4 & 3.17 & 11.00 & 0.79 & $\begin{array}{r}36 . \\
85\end{array}$ & 30.00 & 1.71 & 2.05 & 57 \\
\hline
\end{tabular}




\begin{tabular}{|c|c|c|c|c|c|c|}
\hline \multicolumn{7}{|l|}{ ge } \\
\hline 1 & 3 & 1 & 11.3 & 3 & 4.3 & 14.5 \\
\hline 1 & 3 & 2 & 11.7 & 3 & 4.6 & 15 \\
\hline 1 & 3 & 3 & 12.1 & 3 & 5 & 14 \\
\hline \multicolumn{2}{|c|}{$\begin{array}{l}\text { Averag } \\
\text { e }\end{array}$} & & 11.70 & 3.00 & 4.63 & 14.50 \\
\hline 2 & 1 & 1 & 7 & 5 & 1.6 & 10.3 \\
\hline 2 & 1 & 2 & 8.6 & 5 & 1 & 8.5 \\
\hline 2 & 1 & 3 & 10.2 & 6 & 2.3 & 12 \\
\hline $\begin{array}{l}\text { A } \\
\text { ve } \\
\text { ra } \\
\text { ge }\end{array}$ & & & 8.60 & 5.33 & 1.63 & 10.27 \\
\hline 2 & 2 & 1 & 5.9 & 5 & 1 & 8.5 \\
\hline 2 & 2 & 2 & 7.7 & 6 & 2.5 & 9 \\
\hline 2 & 2 & 3 & 7.9 & 6 & 2.3 & 8 \\
\hline $\begin{array}{l}\text { A } \\
\text { ve } \\
\text { ra } \\
\text { ge }\end{array}$ & & & 7.17 & 5.67 & 1.93 & 8.5 \\
\hline 2 & 3 & 1 & 7.6 & 6 & 1.8 & 7 \\
\hline 2 & 3 & 2 & 6.6 & 5 & 2.2 & 8 \\
\hline 2 & 3 & 3 & 7.6 & 7 & 1.8 & 9 \\
\hline $\begin{array}{l}\text { Av } \\
\text { e }\end{array}$ & & & 6 & 6.00 & 1.93 & 8.00 \\
\hline 3 & 1 & 1 & 13 & 6 & 3 & 12 \\
\hline 3 & 1 & 2 & 12.9 & 6 & 3.6 & 19 \\
\hline 3 & 1 & 3 & 10.9 & 7 & 3.5 & 15 \\
\hline $\begin{array}{l}\text { A } \\
\text { ve } \\
\text { ra } \\
\text { ge }\end{array}$ & & & 12.27 & 6.33 & 3.37 & 15.33 \\
\hline 3 & 2 & 1 & 13 & 5 & 4.2 & 16 \\
\hline 3 & 2 & 2 & 12.6 & 5.5 & 2.6 & 11 \\
\hline
\end{tabular}

\begin{tabular}{|c|c|c|c|c|c|}
\hline & & & & & \\
\hline & & & & & \\
\hline & 46. & & & & \\
\hline 0.47 & 4 & 17 & 2.59 & 2.33 & 57 \\
\hline & 45. & & & & \\
\hline 0.47 & 7 & 16.9 & 1.97 & 1.99 & 59 \\
\hline 0.56 & 45 & 16.9 & 1.11 & 1.34 & 60 \\
\hline 0.5 & $\begin{array}{r}45 . \\
70\end{array}$ & 16.93 & 1.89 & 1.89 & 58.7 \\
\hline & 52. & & & & \\
\hline 0.49 & 7 & 20 & 1.85 & 1.71 & 58 \\
\hline & 55. & & & & \\
\hline 0.42 & 9 & 18 & 2.97 & 1.7 & 59 \\
\hline & 53. & & & & \\
\hline 0.57 & 3 & 22 & 2.41 & 1.85 & 60 \\
\hline 0.49 & $\begin{array}{r}53 . \\
97 \\
\end{array}$ & 20.00 & 2.41 & 1.75 & 59 \\
\hline & 52. & & & & \\
\hline 0.39 & 5 & 30 & 1.86 & 2.47 & 56 \\
\hline & 52. & & & & \\
\hline 0.44 & 1 & 33 & 2.75 & 2.33 & 52 \\
\hline & 52. & & & & \\
\hline 0.46 & 3 & 36 & 3.46 & 2.15 & 59 \\
\hline 0.43 & $\begin{array}{r}52 . \\
30\end{array}$ & 33.00 & 2.69 & 2.32 & 55.7 \\
\hline 0.5 & 53 & 22.5 & 4.88 & 2.58 & 60 \\
\hline & 52. & & & & \\
\hline 0.48 & 2 & 25.3 & 3.88 & 1.88 & 59 \\
\hline & 53. & & & & \\
\hline 0.55 & 8 & 28 & 3.38 & 2.37 & 60 \\
\hline 0.51 & $\begin{array}{r}53 . \\
00\end{array}$ & 25.27 & 4.05 & 2.28 & 59.7 \\
\hline & 59. & & & & \\
\hline 0.42 & 5 & 18 & 1.48 & 2.44 & 50 \\
\hline & 54. & & & & \\
\hline 0.45 & 4 & 20.5 & 1.75 & 3.7 & 49 \\
\hline 0.48 & 58 & 17.5 & 1.42 & 2.65 & 51 \\
\hline & 57. & & & & \\
\hline 0.45 & 3 & 18.67 & 1.55 & 2.93 & 50 \\
\hline & 59. & & & & \\
\hline 0.19 & 4 & 20 & 2.32 & 2.97 & 58 \\
\hline 0.21 & 57. & 19 & 1.89 & 2.8 & 56 \\
\hline
\end{tabular}




\begin{tabular}{|c|c|c|c|c|c|c|c|c|c|c|c|c|}
\hline & & & & & & & & 6 & & & & \\
\hline 3 & 2 & 3 & 12.2 & 6 & 3.3 & 13.5 & 0.18 & $\begin{array}{r}61 . \\
2\end{array}$ & 17.5 & 1.71 & 2.93 & 57 \\
\hline $\begin{array}{l}\text { A } \\
\text { ve } \\
\text { ra } \\
\text { ge }\end{array}$ & & & 12.6 & 5.50 & 3.37 & 13.5 & 0.19 & $\begin{array}{r}59 . \\
4\end{array}$ & 18.83 & 1.97 & 2.9 & 57 \\
\hline 3 & 3 & 1 & 11.5 & 5 & 3.7 & 14 & 0.58 & $\begin{array}{r}65 . \\
3\end{array}$ & 21 & 1.69 & 2.62 & 57 \\
\hline 3 & 3 & 2 & 12.5 & 5 & 3.2 & 16 & 0.32 & 62 & 20 & 2.51 & 2.35 & 59 \\
\hline 3 & 3 & 3 & 13.1 & 7 & 4 & 12 & 0.45 & $\begin{array}{r}68 . \\
6\end{array}$ & 24.5 & 1.4 & 2.43 & 60 \\
\hline $\begin{array}{l}\text { Ave } \\
\text { e }\end{array}$ & & & 12.37 & 5.67 & 3.63 & 14.00 & 0.45 & $\begin{array}{r}65 . \\
30 \\
\end{array}$ & 21.83 & 1.87 & 2.47 & 58.7 \\
\hline 4 & 1 & 1 & 13.1 & 7 & 4.3 & 10 & 0.47 & $\begin{array}{r}26 . \\
65\end{array}$ & 17 & 1.67 & 2.92 & 58 \\
\hline 4 & 1 & 2 & 12.9 & 8 & 5.4 & 17 & 0.52 & $\begin{array}{r}27 . \\
2\end{array}$ & 17.7 & 1.29 & 3.16 & 59 \\
\hline 4 & 1 & 3 & 13.2 & 6 & 4.6 & 16 & 0.42 & $\begin{array}{r}26 . \\
1 \\
\end{array}$ & 18.5 & 1.46 & 1.81 & 60 \\
\hline $\begin{array}{l}\text { A } \\
\text { ve } \\
\text { ra } \\
\text { ge }\end{array}$ & & & 13.07 & 7 & 4.77 & 14.33 & 0.47 & $\begin{array}{r}26 . \\
65\end{array}$ & 17.73 & 1.47 & 2.63 & 59 \\
\hline 4 & 2 & 1 & 12.4 & 9 & 4.3 & 9 & 0.31 & $\begin{array}{r}35 . \\
3\end{array}$ & 19 & 2.02 & 2.41 & 56 \\
\hline 4 & 2 & 2 & 12.6 & 8.5 & 4.5 & 8 & 0.36 & $\begin{array}{r}38 . \\
2 \\
\end{array}$ & 19 & 1.1 & 1.89 & 52 \\
\hline 4 & 2 & 3 & 11.4 & 8 & 3.1 & 10 & 0.33 & $\begin{array}{r}34 . \\
3\end{array}$ & 19 & 1.86 & 2.42 & 59 \\
\hline $\begin{array}{l}\text { A } \\
\text { ve } \\
\text { ra } \\
\text { ge }\end{array}$ & & & 12.13 & 8.5 & 3.97 & 9.00 & 0.33 & $\begin{array}{r}35 . \\
93\end{array}$ & 19.00 & 1.66 & 2.24 & 55.7 \\
\hline 4 & 3 & 1 & 10.7 & 7 & 4.1 & 13.5 & 0.4 & $\begin{array}{r}50 . \\
3\end{array}$ & 22.5 & 1.99 & 2.08 & 60 \\
\hline 4 & 3 & 2 & 13.1 & 7 & 4.8 & 11 & 0.54 & $\begin{array}{r}47 . \\
2\end{array}$ & 22.5 & 2.85 & 2.29 & 59 \\
\hline 4 & 3 & 3 & 13.1 & 6 & 4.5 & 16 & 0.43 & $\begin{array}{r}53 . \\
1\end{array}$ & 25 & 1.25 & 2.27 & 60 \\
\hline $\begin{array}{l}\text { Ave } \\
\text { e }\end{array}$ & & & 12.30 & 6.67 & 4.47 & 13.50 & 0.46 & $\begin{array}{r}50 . \\
20\end{array}$ & 23.33 & 2.03 & 2.21 & 59.7 \\
\hline 5 & 1 & 1 & 13.6 & 6 & 3.3 & 14 & 0.49 & $\begin{array}{r}62 . \\
6\end{array}$ & 21 & 3.34 & 2.9 & 50 \\
\hline 5 & 1 & 2 & 13.1 & 5 & 3 & 10 & 0.57 & $\begin{array}{r}61 . \\
2\end{array}$ & 21 & 2.91 & 3.38 & 49 \\
\hline 5 & 1 & 3 & 12.6 & 5 & 3.2 & 12 & 0.53 & 64 & 23 & 2.72 & 3.26 & 51 \\
\hline
\end{tabular}




\begin{tabular}{|c|c|c|c|c|c|c|c|c|c|c|c|c|}
\hline $\begin{array}{l}\text { A } \\
\text { ve } \\
\text { ra } \\
\text { ge }\end{array}$ & & & 13.1 & 5.33 & 3.17 & 12 & 0.53 & $\begin{array}{r}62 . \\
60\end{array}$ & 21.67 & 2.99 & 3.18 & 50 \\
\hline 5 & 2 & 1 & 14.3 & 5 & 3.6 & 5.8 & \multirow[t]{2}{*}{0.53} & $\begin{array}{r}46 . \\
7\end{array}$ & 23 & 2.53 & 3.53 & 58 \\
\hline 5 & 2 & 2 & 12.9 & 7 & 3.1 & 5.75 & & $\begin{array}{r}55 . \\
7\end{array}$ & 19 & 2.49 & 4.88 & 56 \\
\hline 5 & 2 & 3 & 12.6 & 7 & 3.4 & 6 & 0.51 & $\begin{array}{r}58 . \\
7\end{array}$ & 23 & 4.19 & 3.83 & 57 \\
\hline $\begin{array}{l}\text { A } \\
\text { ve } \\
\text { ra } \\
\text { ge }\end{array}$ & & & 13.27 & 6.33 & 3.37 & 5.85 & & $\begin{array}{r}53 . \\
7\end{array}$ & 21.67 & 3.07 & 4.08 & 57 \\
\hline 5 & 3 & 1 & 12.6 & 9 & 3 & 5.5 & 0.53 & $\begin{array}{r}53 . \\
7\end{array}$ & 23 & 3.04 & 4.07 & 57 \\
\hline 5 & 3 & 2 & 14.1 & 6 & 3.5 & 5 & 0.55 & $\begin{array}{r}53 . \\
5\end{array}$ & 22 & 2.55 & 4.05 & 59 \\
\hline 5 & 3 & 3 & 13.7 & 7 & 3.4 & 6 & 0.58 & $\begin{array}{r}51 . \\
5\end{array}$ & 21 & 2.33 & 3.22 & 60 \\
\hline \multicolumn{2}{|c|}{$\begin{array}{l}\text { Averag } \\
\text { e }\end{array}$} & & 13.47 & 7.33 & 3.30 & 5.50 & 0.52 & $\begin{array}{r}52 . \\
90 \\
\end{array}$ & 22.00 & 2.64 & 3.78 & 58.7 \\
\hline 6 & 1 & 1 & 13.1 & 4 & 4.7 & 12.3 & 0.35 & $\begin{array}{r}61 . \\
9\end{array}$ & 23 & 1.16 & 3.44 & 58 \\
\hline 6 & 1 & 2 & 12.9 & 5 & 4 & 12 & \multirow{2}{*}{$\begin{array}{l}0.44 \\
0.39\end{array}$} & $\begin{array}{r}64 . \\
9\end{array}$ & 23 & 1.64 & 3.6 & 59 \\
\hline 6 & 1 & 3 & 12.8 & 4 & 3.2 & 12.7 & & 68 & 23 & 1.61 & 3.46 & 60 \\
\hline $\begin{array}{l}\text { A } \\
\text { ve } \\
\text { ra } \\
\text { ge }\end{array}$ & & & 12.93 & 4.33 & 3.97 & 12.33 & 0.39 & $\begin{array}{r}64 . \\
93 \\
\end{array}$ & 23.00 & 1.47 & 3.5 & 59 \\
\hline 6 & 2 & 1 & 9.8 & 6 & 3.6 & 12 & \multirow{2}{*}{$\begin{array}{l}0.44 \\
0.41\end{array}$} & $\begin{array}{r}49 . \\
5\end{array}$ & 24 & 2.81 & 4.09 & 56 \\
\hline 6 & 2 & 2 & 10.4 & 5.5 & 4 & 16 & & 52 & 25 & 3.22 & 4.24 & 52 \\
\hline 6 & 2 & 3 & 9.2 & 5 & 4.1 & 14 & 0.39 & $\begin{array}{r}46 . \\
9\end{array}$ & 20 & 2.4 & 3.44 & 59 \\
\hline $\begin{array}{l}\text { A } \\
\text { ve } \\
\text { ra } \\
\text { ge }\end{array}$ & & & 9.8 & 5.5 & 3.9 & 14.00 & 0.41 & $\begin{array}{r}49 . \\
47\end{array}$ & 23 & 2.81 & 3.92 & 55.7 \\
\hline 6 & 3 & 1 & 12.3 & 6 & 3.8 & 18 & 0.33 & $\begin{array}{r}54 . \\
3\end{array}$ & 22 & 1.97 & 2.81 & 60 \\
\hline 6 & 3 & 2 & 13.5 & 4 & 4.5 & 16 & 0.31 & $\begin{array}{r}62 . \\
1\end{array}$ & 23 & 2.7 & 3.59 & 59 \\
\hline 6 & 3 & 3 & 12.2 & 6 & 3.5 & 15 & 0.37 & $\begin{array}{r}58 . \\
2 \\
\end{array}$ & 22.5 & 3.11 & 3.68 & 60 \\
\hline $\begin{array}{l}\text { Av } \\
\text { e }\end{array}$ & & & 12.67 & 5.33 & 3.93 & 16.33 & 0.34 & $\begin{array}{r}58 . \\
20\end{array}$ & 22.50 & 2.59 & 3.36 & 59.7 \\
\hline
\end{tabular}


Table 4: Data of Waterstress

\begin{tabular}{|c|c|c|c|c|c|c|c|c|c|c|c|c|}
\hline $\begin{array}{l}\text { V } \\
\text { ar } \\
\text { ie } \\
\text { ty }\end{array}$ & $\begin{array}{l}\text { Re } \\
\text { pli } \\
\text { cat } \\
\text { ion }\end{array}$ & $\begin{array}{l}\text { No } \\
\text {.of } \\
\text { pla } \\
\text { nts }\end{array}$ & $\begin{array}{l}\text { Pod } \\
\text { lengt } \\
\text { h } \\
(\mathrm{cm}) \\
(\mathrm{PL})\end{array}$ & $\begin{array}{l}\text { No.of } \\
\text { pods/ } \\
\text { plant } \\
\text { (PN) }\end{array}$ & $\begin{array}{l}\text { No.of } \\
\text { seeds/ } \\
\text { pod } \\
\text { (SN) }\end{array}$ & $\begin{array}{l}\text { Seed } \\
\text { weight/ } \\
\text { plant }(g \\
)(\mathrm{SW})\end{array}$ & $\begin{array}{l}\text { Leaf } \\
\text { area } \\
(\mathrm{dm} 2) \\
(\mathrm{LA})\end{array}$ & $\begin{array}{l}\mathbf{R} \\
\mathbf{W} \\
\mathrm{C}( \\
\% \\
)\end{array}$ & $\begin{array}{l}\text { Root } \\
\text { lengt } \\
\text { h } \\
\text { (cm) } \\
(\mathrm{RL})\end{array}$ & $\begin{array}{l}\text { Root } \\
\text { weig } \\
\text { ht(g) } \\
\text { (RW) }\end{array}$ & $\begin{array}{l}\text { Shoot } \\
\text { weight } \\
\text { (g) } \\
\text { (SHW } \\
\text { ) }\end{array}$ & $\begin{array}{l}\text { Mat } \\
\text { urit } \\
\text { y } \\
\text { (Da } \\
\text { ys) }\end{array}$ \\
\hline 1 & 1 & 1 & 12.4 & 2 & 4.3 & 8 & 0.61 & $\begin{array}{r}69 . \\
5\end{array}$ & 23 & 2.21 & 2.95 & 60 \\
\hline 1 & 1 & 2 & 10.2 & 4 & 2.3 & 5 & 0.63 & $\begin{array}{r}65 . \\
4\end{array}$ & 22 & 1.28 & 2.01 & 59 \\
\hline 1 & 1 & 3 & 11.1 & 3 & 3 & 6.5 & 0.62 & $\begin{array}{r}61 . \\
2\end{array}$ & 24 & 1.05 & 2.45 & 60 \\
\hline $\begin{array}{l}\text { A } \\
\text { ve } \\
\text { ra } \\
\text { ge }\end{array}$ & & & 11.23 & 3 & 3.2 & 6.50 & 0.62 & $\begin{array}{r}65 . \\
37\end{array}$ & 23 & 1.51 & 2.47 & 59.7 \\
\hline 1 & 2 & 1 & 9.3 & 3 & 3.3 & 8 & 0.58 & $\begin{array}{r}53 . \\
3\end{array}$ & 22 & 1.16 & 2.51 & 67 \\
\hline 1 & 2 & 2 & 11.2 & 3 & 3.3 & 8 & 0.53 & $\begin{array}{r}54 . \\
8\end{array}$ & 23 & 1 & 2.33 & 66 \\
\hline 1 & 2 & 3 & 8 & 4 & 2.3 & 6 & 0.49 & $\begin{array}{r}56 . \\
3\end{array}$ & 26.5 & 1.26 & 2.16 & 65 \\
\hline $\begin{array}{l}\text { A } \\
\text { ve } \\
\text { ra } \\
\text { ge }\end{array}$ & & & 9.5 & 3.33 & 2.97 & 7.33 & 0.53 & $\begin{array}{r}54 . \\
80\end{array}$ & 23.83 & 1.14 & 2.33 & 66 \\
\hline 1 & 3 & 1 & 12.3 & 3 & 3 & 8 & 0.63 & $\begin{array}{r}41 . \\
5\end{array}$ & 28 & 1.12 & 2.3 & 60 \\
\hline 1 & 3 & 2 & 10.2 & 3 & 2.6 & 5 & 0.51 & $\begin{array}{r}39 . \\
7\end{array}$ & 26 & 1.02 & 2.2 & 61 \\
\hline 1 & 3 & 3 & 11.5 & 2 & 3 & 5 & 0.57 & $\begin{array}{r}44 . \\
7\end{array}$ & 22 & 0.89 & 2.08 & 59 \\
\hline \multicolumn{2}{|c|}{$\begin{array}{l}\text { Averag } \\
\text { e }\end{array}$} & & 11.33 & 2.67 & 2.87 & 6.00 & 0.57 & $\begin{array}{r}41 . \\
97\end{array}$ & 25.33 & 1.01 & 2.19 & 60 \\
\hline 2 & 1 & 1 & 7.2 & 5.5 & 2.5 & 3 & 0.44 & $\begin{array}{r}51 . \\
2\end{array}$ & 21 & 1.81 & 2.72 & 68 \\
\hline 2 & 1 & 2 & 9.1 & 5 & 2.6 & 3 & 0.37 & $\begin{array}{r}46 . \\
5\end{array}$ & 28 & 2.3 & 3.99 & 63 \\
\hline 2 & 1 & 3 & 6.9 & 6 & 1.2 & 3 & 0.51 & 44 & 26 & 1.55 & 1.68 & 64 \\
\hline \multicolumn{2}{|c|}{$\begin{array}{l}\text { Averag } \\
\text { e }\end{array}$} & & 7.73 & 5.50 & 2.1 & 3 & 0.44 & $\begin{array}{r}47 . \\
23\end{array}$ & 25 & 1.89 & 2.80 & 65 \\
\hline 2 & 2 & 1 & 6.5 & 8 & 1.7 & 4 & 0.32 & $\begin{array}{r}36 . \\
7\end{array}$ & 24 & 1.38 & 2.01 & 65 \\
\hline 2 & 2 & 2 & 6.8 & 8 & 1.5 & 4 & 0.3 & $\begin{array}{r}37 . \\
05\end{array}$ & 26 & 2.57 & 3.14 & 62 \\
\hline 2 & 2 & 3 & 8.4 & 8 & 2.7 & 4 & 0.28 & 37. & 26 & 1.68 & 3.12 & 61 \\
\hline
\end{tabular}




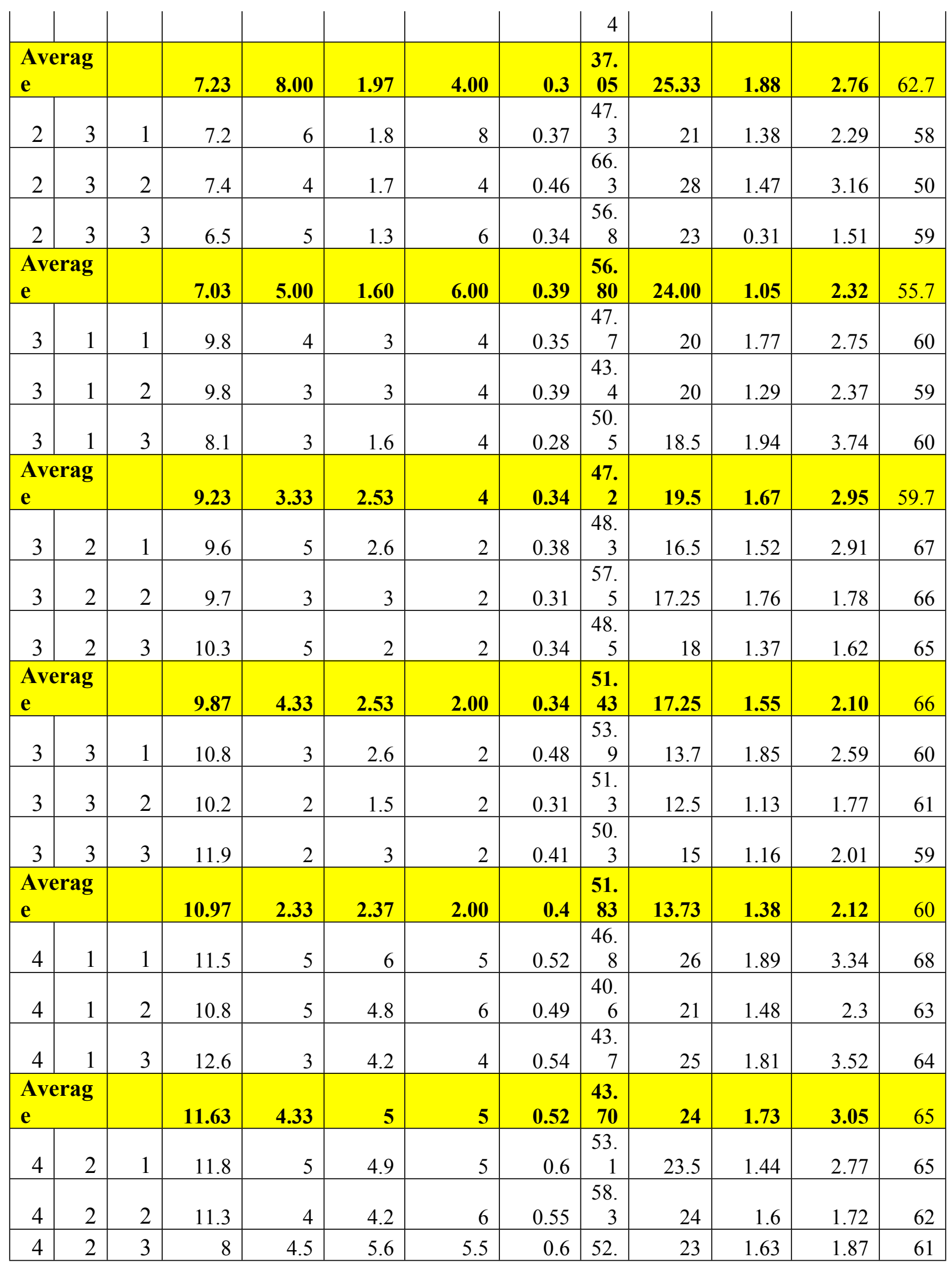




\begin{tabular}{|c|c|c|c|c|c|c|c|c|c|c|c|c|}
\hline & & & & & & & & 1 & & & & \\
\hline $\begin{array}{l}\text { Ave } \\
\text { e }\end{array}$ & & & 10.37 & 4.50 & 4.90 & 5.50 & 0.58 & $\begin{array}{r}54 . \\
5\end{array}$ & 23.50 & 1.56 & 2.12 & 62.7 \\
\hline 4 & 3 & 1 & 11.2 & 4 & 4.5 & 3 & 0.41 & $\begin{array}{r}53 . \\
7\end{array}$ & 24 & 1.31 & 2.46 & 58 \\
\hline 4 & 3 & 2 & 10.9 & 3 & 4.5 & 4 & 0.53 & $\begin{array}{r}59 . \\
3\end{array}$ & 22 & 1.88 & 2.7 & 50 \\
\hline 4 & 3 & 3 & 10.3 & 2 & 4 & 3.5 & 0.47 & $\begin{array}{r}63 . \\
3\end{array}$ & 23 & 1.59 & 2.58 & 59 \\
\hline $\begin{array}{l}\text { Ave } \\
\text { e }\end{array}$ & & & 10.80 & 3.00 & 4.33 & 3.50 & 0.47 & $\begin{array}{r}58 . \\
77 \\
\end{array}$ & 23.00 & 1.59 & 2.58 & 55.7 \\
\hline 5 & 1 & 1 & 12.6 & 4 & 2.3 & 2 & 0.49 & $\begin{array}{r}44 . \\
8\end{array}$ & 24 & 1.42 & 2.63 & 60 \\
\hline 5 & 1 & 2 & 12.3 & 5 & 2.8 & 2.5 & 0.47 & $\begin{array}{r}48 . \\
4\end{array}$ & 20 & 1.09 & 2.1 & 59 \\
\hline 5 & 1 & 3 & 12.3 & 5 & 2.6 & 3 & 0.51 & $\begin{array}{r}53 . \\
9 \\
\end{array}$ & 23.5 & 1.16 & 1.56 & 60 \\
\hline $\begin{array}{l}\text { Ave } \\
\text { e }\end{array}$ & & & 12.4 & 4.67 & 2.57 & 2.5 & 0.49 & $\begin{array}{r}49 . \\
03 \\
\end{array}$ & 22.5 & 1.22 & 2.10 & 59.7 \\
\hline 5 & 2 & 1 & 13.3 & 4 & 3 & 6 & 0.4 & $\begin{array}{r}44 . \\
5\end{array}$ & 24 & 1.67 & 3.01 & 67 \\
\hline 5 & 2 & 2 & 13.3 & 5 & 3 & 5 & 0.59 & $\begin{array}{r}51 . \\
2\end{array}$ & 22.5 & 1.41 & 2.58 & 66 \\
\hline 5 & 2 & 3 & 11.9 & 6 & 3 & 4 & 0.52 & $\begin{array}{r}44 . \\
9 \\
\end{array}$ & 22.5 & 1.36 & 2.73 & 65 \\
\hline $\begin{array}{l}\text { Ave } \\
\text { e }\end{array}$ & & & 12.83 & 5 & 3.00 & 5.00 & 0.50 & $\begin{array}{r}46 . \\
87 \\
\end{array}$ & 23 & 1.48 & 2.77 & 66 \\
\hline 5 & 3 & 1 & 14.8 & 6 & 3.6 & 5 & 0.45 & $\begin{array}{r}51 . \\
1 \\
\end{array}$ & 27 & 1.49 & 3.05 & 60 \\
\hline 5 & 3 & 2 & 11 & 7 & 2.3 & 4.5 & 0.42 & $\begin{array}{r}53 . \\
5\end{array}$ & 27 & 1.04 & 2.95 & 61 \\
\hline 5 & 3 & 3 & 12 & 5 & 2.4 & 4 & 0.53 & $\begin{array}{r}55 . \\
9\end{array}$ & 27 & 1.21 & 2.74 & 59 \\
\hline $\begin{array}{l}\text { Ave } \\
\text { e }\end{array}$ & & & 12.60 & 6.00 & 2.77 & 4.50 & 0.47 & $\begin{array}{r}53 . \\
50\end{array}$ & 27.00 & 1.25 & 2.91 & 60 \\
\hline 6 & 1 & 1 & 12.6 & 5 & 2.4 & 7 & 0.47 & $\begin{array}{r}56 . \\
4 \\
\end{array}$ & 22 & 1.32 & 2.36 & 68 \\
\hline 6 & 1 & 2 & 14.1 & 6 & 2.3 & 5 & 0.49 & $\begin{array}{r}57 . \\
1\end{array}$ & 25 & 1.65 & 2.55 & 63 \\
\hline 6 & 1 & 3 & 11.1 & 4 & 3.5 & 6 & 0.52 & $\begin{array}{r}56 . \\
5 \\
\end{array}$ & 22 & 1.47 & 2.47 & 64 \\
\hline $\begin{array}{l}\text { Ave } \\
\text { e }\end{array}$ & & & 12.60 & 5 & 2.73 & 6 & 0.49 & $\begin{array}{r}56 . \\
67 \\
\end{array}$ & 23 & 1.48 & 2.46 & 65 \\
\hline 6 & 2 & 1 & 7.6 & 5 & 2.7 & 4 & 0.34 & $\begin{array}{r}66 . \\
4\end{array}$ & 22 & 1.36 & 1.58 & 65 \\
\hline 6 & 2 & 2 & 8.5 & 6 & 2.6 & 6 & 0.29 & $\begin{array}{r}65 . \\
9\end{array}$ & 28 & 1.88 & 2.9 & 62 \\
\hline 6 & 2 & 3 & 9.4 & 5 & 3 & 6 & 0.32 & 56 & 25 & 2.37 & 2.7 & 61 \\
\hline
\end{tabular}




\begin{tabular}{|c|c|c|c|c|c|c|c|c|c|c|c|c|}
\hline \multicolumn{2}{|c|}{$\begin{array}{l}\text { Averag } \\
\text { e }\end{array}$} & & 8.5 & 5.33 & 2.77 & 5.33 & 0.32 & $\begin{array}{r}62 . \\
77\end{array}$ & 25 & 1.87 & 2.39 & 62.7 \\
\hline 6 & 3 & 1 & 7.8 & 5 & 2.2 & 8 & 0.47 & $\begin{array}{r}55 . \\
9\end{array}$ & 22 & 1.66 & 4.18 & 58 \\
\hline 6 & 3 & 2 & 7.9 & 4.5 & 2.8 & 5 & 0.56 & $\begin{array}{r}65 . \\
4\end{array}$ & 21 & 2.36 & 4.21 & 50 \\
\hline 6 & 3 & 3 & 7.85 & 4 & 3 & 6.5 & 0.51 & $\begin{array}{r}59 . \\
8 \\
\end{array}$ & 24 & 2.55 & 4.15 & 59 \\
\hline \multicolumn{2}{|c|}{$\begin{array}{l}\text { Averag } \\
\text { e }\end{array}$} & & 7.85 & 4.50 & 2.67 & 6.50 & 0.51 & $\begin{array}{r}60 . \\
37 \\
\end{array}$ & 22.33 & 2.19 & 4.18 & 55.7 \\
\hline
\end{tabular}

Table 5: Standard Deviation

\begin{tabular}{|c|c|c|c|c|c|c|c|c|c|c|c|c|}
\hline TRT & VAR & REP & PL & PN & SN & SW & LA & RWC & RL & RW & SHW & Maturity \\
\hline 1 & 1 & 1 & 12.60 & 3.99 & 4.23 & 12.70 & 0.37 & 46.03 & 28.90 & 2.43 & 2.69 & 50 \\
\hline 1 & 1 & 2 & 12.97 & 4 & 3.17 & 11.00 & 0.79 & 45.86 & 30.00 & 2.16 & 2.68 & 49 \\
\hline 1 & 1 & 3 & 11.70 & 3.99 & 4.63 & 14.50 & 0.50 & 45.7 & 27.80 & 2.29 & 2.67 & 51 \\
\hline \multicolumn{3}{|c|}{ SD } & 0.65 & 0.01 & 0.76 & 1.75 & 0.22 & 0.17 & 1.10 & 0.14 & 0.01 & 1.00 \\
\hline 1 & 2 & 1 & 8.60 & 5.33 & 1.63 & 10.27 & 0.49 & 53.97 & 29.14 & 2.41 & 2.30 & 58 \\
\hline 1 & 2 & 2 & 8.24 & 5.67 & 1.93 & 8.5 & 0.43 & 52.30 & 33.00 & 2.69 & 2.32 & 56 \\
\hline 1 & 2 & 3 & 7.88 & 6 & 1.93 & 8.00 & 0.51 & 53.00 & 31.07 & 2.55 & 2.31 & 57 \\
\hline \multicolumn{3}{|c|}{ SD } & 0.36 & 0.33 & 0.17 & 1.19 & 0.04 & 0.84 & 1.93 & 0.14 & 0.01 & 1.00 \\
\hline 1 & 3 & 1 & 12.27 & 6.33 & 3.37 & 15.33 & 0.45 & 57.3 & 18.67 & 1.96 & 2.93 & 58 \\
\hline 1 & 3 & 2 & 12.6 & 5.50 & 3.37 & 13.5 & 0.19 & 59.4 & 18.83 & 1.97 & 2.90 & 56 \\
\hline 1 & 3 & 3 & 12.37 & 5.67 & 3.63 & 14 & 0.45 & 65.3 & 21.83 & 1.96 & 2.92 & 57 \\
\hline \multicolumn{3}{|c|}{ SD } & 0.17 & 0.44 & 0.15 & 0.95 & 0.15 & 4.15 & 1.78 & 0.01 & 0.02 & 1.00 \\
\hline 1 & 4 & 1 & 13.07 & 7 & 4.77 & 14.33 & 0.47 & 43.06 & 17.73 & 2.02 & 2.63 & 58 \\
\hline 1 & 4 & 2 & 12.13 & 8.5 & 3.97 & 13.90 & 0.33 & 46.63 & 19.00 & 2.01 & 2.53 & 59 \\
\hline 1 & 4 & 3 & 12.3 & 6.67 & 4.47 & 13.50 & 0.46 & 50.2 & 18.00 & 2.03 & 2.58 & 60 \\
\hline \multicolumn{3}{|c|}{ SD } & 0.50 & 0.98 & 0.40 & 0.42 & 0.08 & 3.57 & 0.67 & 0.01 & 0.05 & 1 \\
\hline 1 & 5 & 1 & 13.10 & 5.33 & 3.17 & 5.67 & 0.53 & 62.60 & 21.67 & 2.99 & 3.93 & 56 \\
\hline 1 & 5 & 2 & 13.27 & 6.33 & 3.37 & 5.85 & 0.53 & 53.7 & 21.67 & 3.07 & 4.08 & 52 \\
\hline 1 & 5 & 3 & 13.47 & 7.33 & 3.30 & 5.50 & 0.52 & 52.9 & 22.0 & 2.64 & 4.005 & 59 \\
\hline \multicolumn{3}{|c|}{ SD } & 0.18 & 1.00 & 0.10 & 0.18 & 0.01 & 5.38 & 0.2 & 0.23 & 0.08 & 3.51 \\
\hline 1 & 6 & 1 & 12.93 & 4.33 & 3.97 & 13.50 & 0.39 & 64.93 & 23.00 & 2.7 & 3.50 & 60 \\
\hline 1 & 6 & 2 & 9.8 & 5.5 & 3.9 & 14.00 & 0.41 & 63.24 & 23 & 2.81 & 3.92 & 59 \\
\hline 1 & 6 & 3 & 12.67 & 5.33 & 3.93 & 16.33 & 0.34 & 61.56 & 22.50 & 2.59 & 3.36 & 60 \\
\hline \multicolumn{3}{|c|}{ SD } & 1.74 & 0.63 & 0.03 & 1.51 & 0.04 & 1.69 & 0.29 & 0.11 & 0.29 & 0.58 \\
\hline 2 & 1 & 1 & 11.23 & 3 & 3.2 & 6.50 & 0.62 & 48.38 & 23 & 1.51 & 2.47 & 60 \\
\hline 2 & 1 & 2 & 9.5 & 3.33 & 2.97 & 7.33 & 0.53 & 45.17 & 23.83 & 1.14 & 2.33 & 59 \\
\hline 2 & 1 & 3 & 11.33 & 2.67 & 2.87 & 6.92 & 0.57 & 41.97 & 25.33 & 1.01 & 2.19 & 60 \\
\hline \multicolumn{3}{|c|}{ SD } & 1.03 & 0.33 & 0.17 & 0.42 & 0.05 & 3.21 & 1.18 & 0.26 & 0.14 & 0.58 \\
\hline 2 & 2 & 1 & 7.73 & 5.50 & 2.1 & 3 & 0.44 & 39.59 & 25 & 1.89 & 2.80 & 67 \\
\hline
\end{tabular}




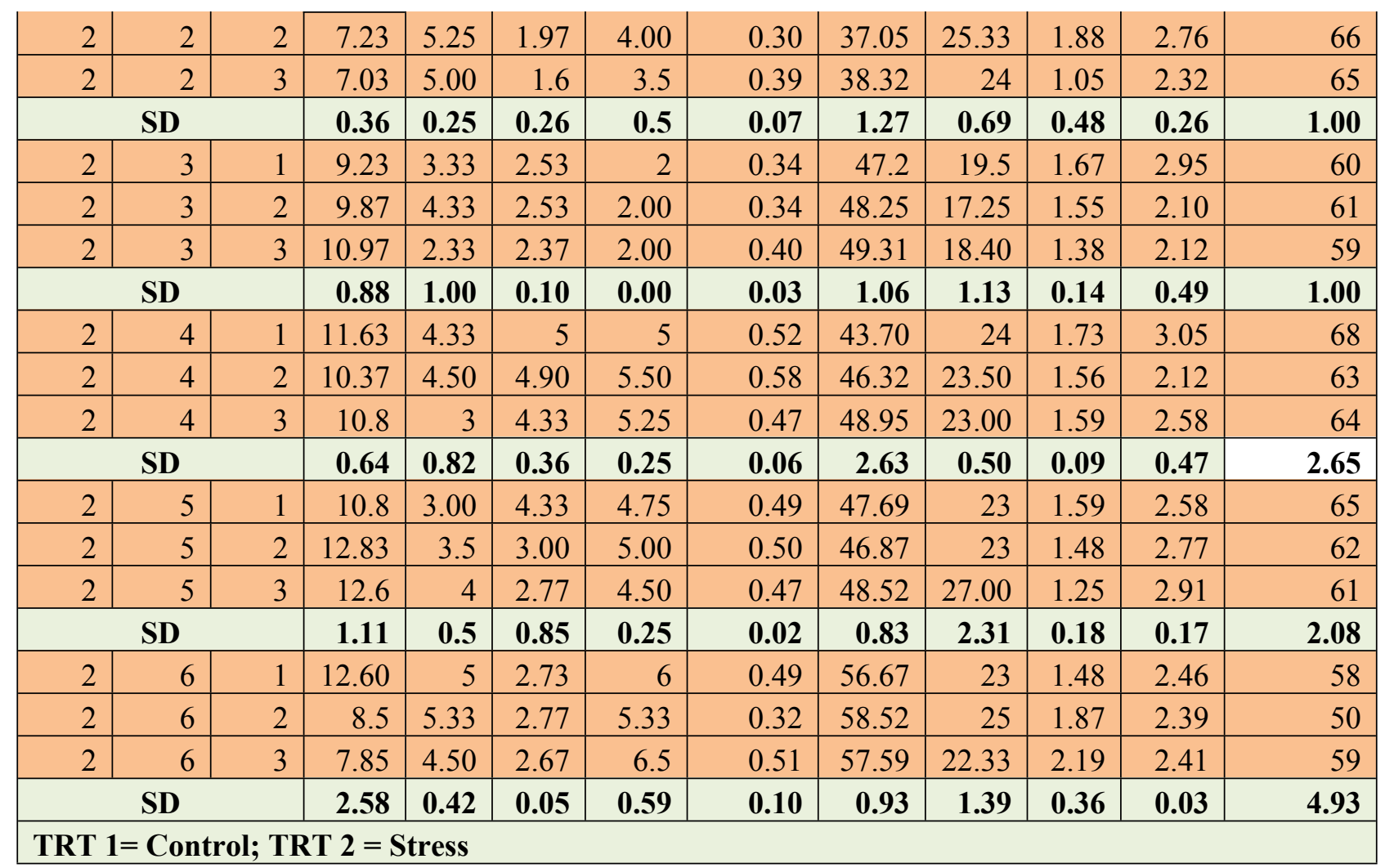

Table 6: DSI Data

\begin{tabular}{|c|c|c|c|c|c|c|c|c|c|c|c|c|c|c|c|c|}
\hline \multirow[t]{2}{*}{ Sl.No } & \multirow[t]{2}{*}{$\begin{array}{l}\text { Genot } \\
\text { ypes }\end{array}$} & \multicolumn{3}{|c|}{$\begin{array}{c}\text { Leaf } \\
\text { area[LA] } \\
(\operatorname{dm} 2) \\
\end{array}$} & \multicolumn{3}{|c|}{$\begin{array}{c}\text { Shoot } \\
\text { weight (g) }\end{array}$} & \multicolumn{3}{|c|}{$\begin{array}{c}\text { Root } \\
\text { weight (g) }\end{array}$} & \multicolumn{3}{|c|}{$\begin{array}{c}\text { Relative water } \\
\text { content[RWC] } \\
(\%)\end{array}$} & \multicolumn{3}{|c|}{$\begin{array}{c}\text { Seed } \\
\text { weight (g) }\end{array}$} \\
\hline & & $\begin{array}{l}\text { D } \\
\text { S }\end{array}$ & $\begin{array}{l}\mathbf{N} \\
\mathbf{S}\end{array}$ & $\begin{array}{l}\text { DS } \\
\text { I }\end{array}$ & $\begin{array}{l}\text { D } \\
\text { S }\end{array}$ & $\begin{array}{l}\mathbf{N} \\
\mathbf{S}\end{array}$ & $\begin{array}{l}\text { D } \\
\text { SI }\end{array}$ & $\begin{array}{l}\text { D } \\
\text { S }\end{array}$ & $\begin{array}{l}\mathbf{N} \\
\mathbf{S}\end{array}$ & $\begin{array}{l}\text { D } \\
\text { SI }\end{array}$ & DS & NS & DSI & $\begin{array}{l}\text { D } \\
\text { S }\end{array}$ & $\begin{array}{l}\mathbf{N} \\
\mathbf{S}\end{array}$ & $\begin{array}{l}\text { D } \\
\text { SI }\end{array}$ \\
\hline 1 & $\begin{array}{l}\text { Orey } \\
\text { serbu }\end{array}$ & $\begin{array}{r}0 . \\
55 \\
\end{array}$ & $\begin{array}{r}0 . \\
75\end{array}$ & $\begin{array}{r}0.1 \\
4 \\
\end{array}$ & $\begin{array}{r}6 . \\
91 \\
\end{array}$ & $\begin{array}{r}12 \\
.7 \\
3 \\
\end{array}$ & $\begin{array}{c}- \\
0 . \\
32\end{array}$ & $\begin{array}{l}1 . \\
2 \\
2\end{array}$ & $\begin{array}{r}2 . \\
2 \\
9 \\
\end{array}$ & $\begin{array}{c}0 . \\
16\end{array}$ & $\begin{array}{r}45.1 \\
7 \\
\end{array}$ & $\begin{array}{r}45.8 \\
6 \\
\end{array}$ & -0.14 & $\begin{array}{r}5 . \\
7 \\
7\end{array}$ & $\begin{array}{r}12 \\
.7 \\
3 \\
\end{array}$ & $\begin{array}{r} \\
0 . \\
19\end{array}$ \\
\hline 2 & $\begin{array}{l}\text { Yadhip } \\
\text { a orey }\end{array}$ & $\begin{array}{r}0 . \\
34 \\
\end{array}$ & $\begin{array}{r}0 . \\
5 \\
\end{array}$ & 0.3 & $\begin{array}{r}3 . \\
5\end{array}$ & $\begin{array}{r}8 . \\
92 \\
\end{array}$ & $\begin{array}{r}0 . \\
04\end{array}$ & $\begin{array}{r}1 . \\
6\end{array}$ & $\begin{array}{r}2 . \\
5 \\
5\end{array}$ & $\begin{array}{r}0 . \\
02\end{array}$ & $\begin{array}{r}38.3 \\
2 \\
\end{array}$ & $\begin{array}{r}53.0 \\
9 \\
\end{array}$ & 0.16 & $\begin{array}{r}4 . \\
3 \\
3\end{array}$ & $\begin{array}{r}8 . \\
92\end{array}$ & $\begin{array}{r}0 . \\
27\end{array}$ \\
\hline 3 & $\begin{array}{l}\text { Orey } \\
\text { brokch } \\
\text { ilu }\end{array}$ & $\begin{array}{r}0 . \\
34 \\
\end{array}$ & $\begin{array}{r}0 . \\
45 \\
\end{array}$ & $\begin{array}{r}0.1 \\
2 \\
\end{array}$ & 2 & $\begin{array}{r}14 \\
.2 \\
7 \\
\end{array}$ & $\begin{array}{r}0 . \\
65 \\
\end{array}$ & $\begin{array}{r}1 . \\
5 \\
3\end{array}$ & $\begin{array}{r}1 . \\
9 \\
6\end{array}$ & $\begin{array}{r}- \\
0 . \\
42 \\
\end{array}$ & $\begin{array}{r}48.2 \\
5 \\
\end{array}$ & $\begin{array}{r}60.6 \\
6 \\
\end{array}$ & 0.07 & 2 & $\begin{array}{r}14 \\
.2 \\
7 \\
\end{array}$ & $\begin{array}{r}0 . \\
63 \\
\end{array}$ \\
\hline 4 & $\begin{array}{l}\text { Orey } \\
\text { regtang }\end{array}$ & $\begin{array}{r}0 . \\
55 \\
\end{array}$ & $\begin{array}{r}0 . \\
46\end{array}$ & $\begin{array}{r}- \\
0.3 \\
9\end{array}$ & $\begin{array}{c}5 . \\
25\end{array}$ & $\begin{array}{r}13 \\
.9 \\
1 \\
\end{array}$ & $\begin{array}{r}0 . \\
07\end{array}$ & $\begin{array}{l}1 . \\
6 \\
2\end{array}$ & $\begin{array}{r}2 . \\
0 \\
2\end{array}$ & $\begin{array}{r}- \\
0 . \\
25\end{array}$ & $\begin{array}{r}46.3 \\
2 \\
\end{array}$ & $\begin{array}{r}46.6 \\
3 \\
\end{array}$ & -0.15 & $\begin{array}{r}4 . \\
6 \\
6\end{array}$ & $\begin{array}{r}13 \\
.9 \\
1 \\
\end{array}$ & $\begin{array}{r}0 . \\
12 \\
\end{array}$ \\
\hline 5 & $\begin{array}{l}\text { Kerong } \\
\text { ree } \\
\text { orey }\end{array}$ & $\begin{array}{r}0 . \\
48 \\
\end{array}$ & $\begin{array}{r}0 . \\
52 \\
\end{array}$ & $\begin{array}{r}- \\
0.0 \\
7\end{array}$ & $\begin{array}{r}4 . \\
75 \\
\end{array}$ & $\begin{array}{r}5 . \\
67 \\
\end{array}$ & $\begin{array}{l}- \\
04\end{array}$ & $\begin{array}{l}1 \\
4 \\
4\end{array}$ & $\begin{array}{r}2 . \\
9 \\
\end{array}$ & $\begin{array}{r}0 . \\
22 \\
\end{array}$ & $\begin{array}{r}47.6 \\
9 \\
\end{array}$ & 56.4 & 0.02 & $\begin{array}{r}4 . \\
3 \\
3 \\
\end{array}$ & $\begin{array}{r}5 . \\
67 \\
\end{array}$ & $\begin{array}{r}3 . \\
44 \\
\end{array}$ \\
\hline
\end{tabular}




\begin{tabular}{|c|c|c|c|c|c|c|c|c|c|c|c|c|c|c|c|c|}
\hline 6 & $\begin{array}{l}\text { Brokpa } \\
\text { li }\end{array}$ & $\begin{array}{r}0 . \\
36\end{array}$ & $\begin{array}{r}0 . \\
4\end{array}$ & $\begin{array}{r}0.0 \\
4\end{array}$ & $\begin{array}{r}5 . \\
94\end{array}$ & $\begin{array}{r}14 \\
.6 \\
1\end{array}$ & $\begin{array}{r}5 . \\
9\end{array}$ & $\begin{array}{c}1 . \\
8 \\
4\end{array}$ & $\begin{array}{r}2 . \\
7\end{array}$ & $\begin{array}{c}0 . \\
06\end{array}$ & $\begin{array}{r}57.5 \\
9\end{array}$ & $\begin{array}{r}63.2 \\
4\end{array}$ & -0.05 & $\begin{array}{c}5 \\
9 \\
4\end{array}$ & $\begin{array}{r}14 \\
.6 \\
1\end{array}$ & $\begin{array}{c}- \\
0 . \\
07\end{array}$ \\
\hline $\begin{array}{l}\text { Gran } \\
\text { d } \\
\text { Mean }\end{array}$ & & $\begin{array}{r}0 . \\
44\end{array}$ & $\begin{array}{r}0 . \\
51\end{array}$ & $\begin{array}{r}0.0 \\
1\end{array}$ & $\begin{array}{r}4 . \\
73\end{array}$ & $\begin{array}{r}11 \\
.6 \\
9\end{array}$ & $\begin{array}{r}0 . \\
88\end{array}$ & $\begin{array}{r}1 . \\
5 \\
4\end{array}$ & 2. & $\begin{array}{r}- \\
0 . \\
05 \\
5\end{array}$ & $\begin{array}{r}47.2 \\
2\end{array}$ & $\begin{array}{r}54.3 \\
1\end{array}$ & 0.015 & $\begin{array}{r}4 . \\
5 \\
1\end{array}$ & $\begin{array}{r}11 \\
.6 \\
9\end{array}$ & $\begin{array}{r}0 . \\
61\end{array}$ \\
\hline
\end{tabular}

\begin{tabular}{|c|c|c|c|c|c|c|c|c|c|c|c|c|}
\hline \multirow[t]{2}{*}{$\begin{array}{l}\text { Genot } \\
\text { ypes }\end{array}$} & \multicolumn{3}{|c|}{$\begin{array}{c}\text { Pod } \\
\text { number } \\
\end{array}$} & \multicolumn{3}{|c|}{ Pod length } & \multicolumn{3}{|c|}{$\begin{array}{c}\text { Seed } \\
\text { number }\end{array}$} & \multicolumn{3}{|c|}{ Root length } \\
\hline & $\begin{array}{l}\text { D } \\
\text { S }\end{array}$ & $\begin{array}{l}\mathbf{N} \\
\mathbf{S}\end{array}$ & $\begin{array}{l}\text { DS } \\
\text { I }\end{array}$ & $\begin{array}{l}\text { D } \\
\text { S }\end{array}$ & $\begin{array}{l}\mathbf{N} \\
\mathbf{S}\end{array}$ & $\begin{array}{l}\text { D } \\
\text { SI }\end{array}$ & $\begin{array}{l}\text { D } \\
\text { S }\end{array}$ & $\begin{array}{l}\mathbf{N} \\
\mathbf{S}\end{array}$ & $\begin{array}{l}\text { D } \\
\text { SI }\end{array}$ & DS & NS & DSI \\
\hline $\begin{array}{l}\text { Orey } \\
\text { serbu }\end{array}$ & 3 & $\begin{array}{r}3 . \\
99\end{array}$ & $\begin{array}{r}- \\
0.0 \\
9\end{array}$ & $\begin{array}{r}10 \\
.6 \\
8\end{array}$ & $\begin{array}{r}12 \\
.4 \\
2\end{array}$ & $\begin{array}{c}- \\
0 . \\
01\end{array}$ & $\begin{array}{r}3 . \\
0 \\
1\end{array}$ & $\begin{array}{r}4 . \\
0 \\
1\end{array}$ & $\begin{array}{r}0 . \\
13\end{array}$ & $\begin{array}{r}24.0 \\
5\end{array}$ & 28.9 & 0.14 \\
\hline $\begin{array}{l}\text { Yadhip } \\
\text { a orey }\end{array}$ & $\begin{array}{r}5 . \\
25\end{array}$ & $\begin{array}{r}5 . \\
66\end{array}$ & $\begin{array}{r}- \\
0.3 \\
4\end{array}$ & $\begin{array}{r}7 . \\
33\end{array}$ & $\begin{array}{r}8 . \\
24\end{array}$ & $\begin{array}{c}- \\
04 \\
04\end{array}$ & $\begin{array}{l}1 . \\
8 \\
9\end{array}$ & $\begin{array}{l}1 . \\
8 \\
3\end{array}$ & $\begin{array}{c}- \\
0 . \\
18\end{array}$ & $\begin{array}{r}24.7 \\
7\end{array}$ & $\begin{array}{r}31.0 \\
7\end{array}$ & 0.17 \\
\hline $\begin{array}{l}\text { Orey } \\
\text { brokch } \\
\text { ilu }\end{array}$ & $\begin{array}{r}3 . \\
33\end{array}$ & $\begin{array}{r}5 . \\
83\end{array}$ & $\begin{array}{r}0.1 \\
7\end{array}$ & $\begin{array}{r}10 \\
.0 \\
2\end{array}$ & $\begin{array}{r}12 \\
.4 \\
1\end{array}$ & $\begin{array}{c}0 . \\
05\end{array}$ & $\begin{array}{r}2 . \\
4 \\
7\end{array}$ & $\begin{array}{l}3 . \\
4 \\
5\end{array}$ & $\begin{array}{c}0 . \\
17\end{array}$ & $\begin{array}{r}18.3 \\
8\end{array}$ & $\begin{array}{r}19.7 \\
7\end{array}$ & 0.04 \\
\hline $\begin{array}{l}\text { Orey } \\
\text { regtang }\end{array}$ & $\begin{array}{r}3 . \\
94\end{array}$ & $\begin{array}{r}7 . \\
39\end{array}$ & $\begin{array}{r}0.2 \\
2\end{array}$ & $\begin{array}{r}10 \\
.9 \\
3\end{array}$ & $\begin{array}{r}12 \\
.5\end{array}$ & $\begin{array}{c}- \\
0 . \\
02\end{array}$ & $\begin{array}{r}4 . \\
7 \\
4\end{array}$ & $\begin{array}{r}4 . \\
4\end{array}$ & $\begin{array}{c}- \\
0 . \\
23\end{array}$ & 23.5 & $\begin{array}{r}18.2 \\
4\end{array}$ & -0.32 \\
\hline $\begin{array}{l}\text { Kerong } \\
\text { ree } \\
\text { orey }\end{array}$ & $\begin{array}{r}3 . \\
5\end{array}$ & $\begin{array}{r}6 . \\
33\end{array}$ & $\begin{array}{r}0.1 \\
9\end{array}$ & $\begin{array}{r}12 \\
.0 \\
7\end{array}$ & $\begin{array}{r}13 \\
.2 \\
8\end{array}$ & $\begin{array}{c}- \\
06\end{array}$ & $\begin{array}{l}3 . \\
3 \\
6\end{array}$ & $\begin{array}{r}3 . \\
2 \\
8\end{array}$ & $\begin{array}{c}- \\
0 . \\
17\end{array}$ & $\begin{array}{r}24.3 \\
3\end{array}$ & $\begin{array}{r}21.7 \\
8\end{array}$ & -0.15 \\
\hline $\begin{array}{l}\text { Brokpa } \\
\text { li }\end{array}$ & $\begin{array}{r}4 . \\
94\end{array}$ & $\begin{array}{r}5 . \\
05\end{array}$ & $\begin{array}{r}- \\
0.4 \\
1\end{array}$ & $\begin{array}{l}9 . \\
65\end{array}$ & $\begin{array}{r}11 \\
.8\end{array}$ & $\begin{array}{c}0 . \\
03\end{array}$ & $\begin{array}{r}2 . \\
7 \\
2\end{array}$ & $\begin{array}{r}3 . \\
9 \\
3\end{array}$ & $\begin{array}{r}0 . \\
2\end{array}$ & $\begin{array}{r}23.4 \\
4\end{array}$ & $\begin{array}{r}22.8 \\
3\end{array}$ & -0.05 \\
\hline & $\begin{array}{r}3 . \\
99\end{array}$ & $\begin{array}{r}5 . \\
71\end{array}$ & $\begin{array}{r}- \\
0.0 \\
4\end{array}$ & $\begin{array}{r}10 \\
.1 \\
1\end{array}$ & $\begin{array}{r}11 \\
.7 \\
8\end{array}$ & $\begin{array}{r}0 . \\
00 \\
8\end{array}$ & $\begin{array}{r}3 . \\
0 \\
3\end{array}$ & $\begin{array}{l}3 . \\
4 \\
8\end{array}$ & 01 & $\begin{array}{r}23.0 \\
8\end{array}$ & $\begin{array}{r}23.7 \\
7\end{array}$ & $\begin{array}{r}- \\
0.028\end{array}$ \\
\hline
\end{tabular}

Table 7: Rank of DSI

Drought susceptibity index (DSI)

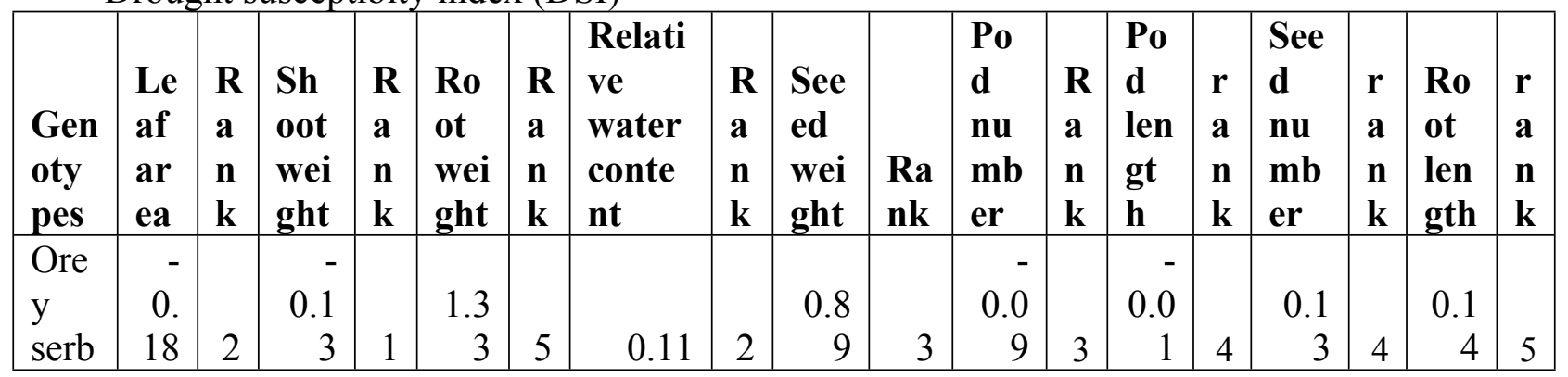




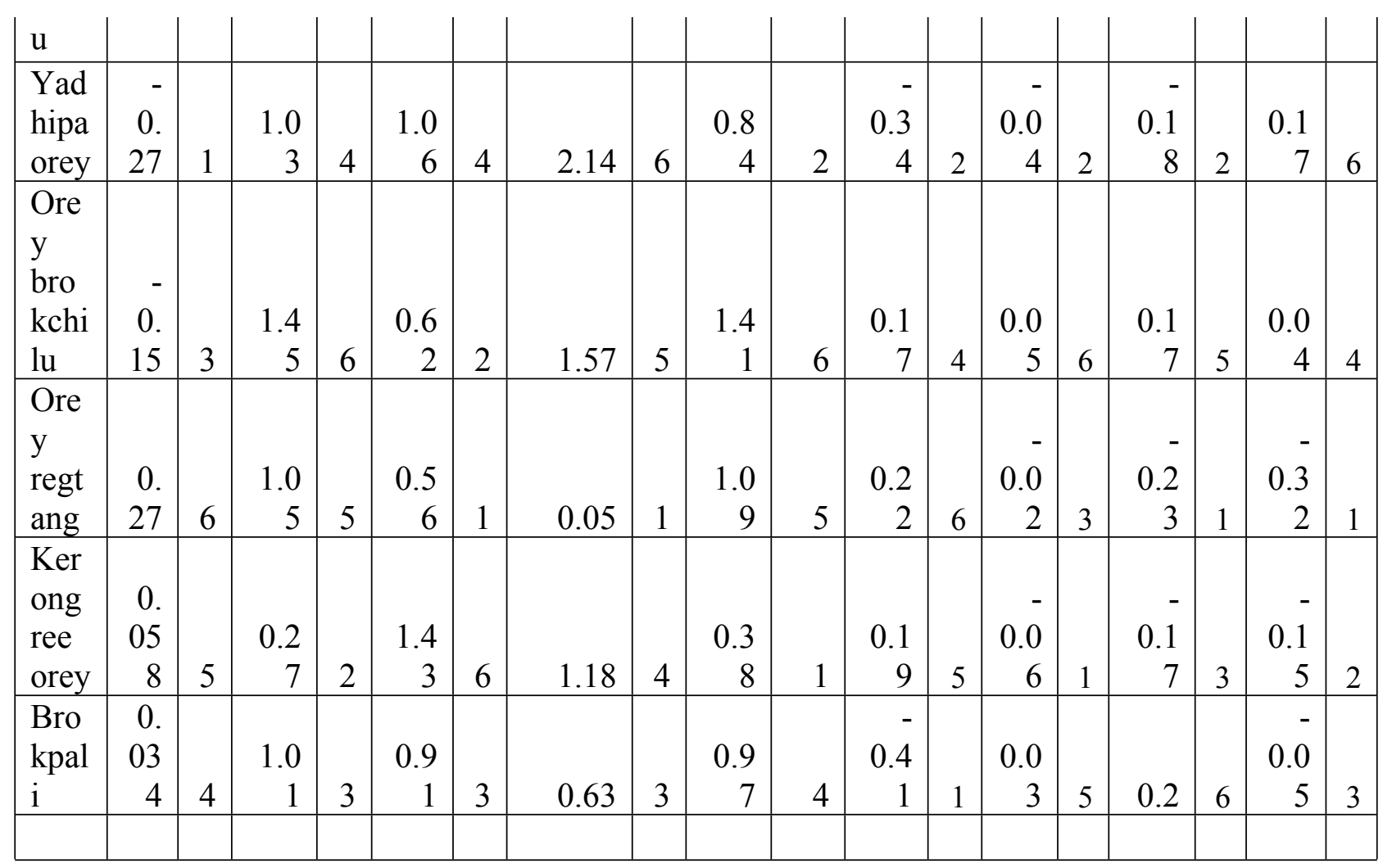

\begin{tabular}{|c|c|c|c|c|c|c|c|c|c|c|c|}
\hline $\begin{array}{l}\text { Gen } \\
\text { oty } \\
\text { pes }\end{array}$ & $\begin{array}{l}\mathbf{L} \\
\mathbf{A}\end{array}$ & $\begin{array}{l}\mathbf{S} \\
\mathbf{H} \\
\mathbf{W} \\
\end{array}$ & $\begin{array}{l}\mathbf{R} \\
\mathbf{W} \\
\end{array}$ & $\begin{array}{l}\mathbf{R} \\
\mathbf{W} \\
\mathbf{C}\end{array}$ & $\begin{array}{c}\mathbf{S} \\
\mathbf{W}\end{array}$ & $\begin{array}{l}\mathbf{P} \\
\mathbf{N}\end{array}$ & PL & $\begin{array}{l}\mathbf{S} \\
\mathbf{N} \\
\end{array}$ & RL & $\begin{array}{l}\text { Ra } \\
\text { nk } \\
\text { su } \\
\text { m }\end{array}$ & $\begin{array}{l}\text { Ra } \\
\text { nk }\end{array}$ \\
\hline $\begin{array}{l}\text { Ore } \\
\text { y } \\
\text { serb } \\
\text { u }\end{array}$ & 2 & 1 & 5 & 2 & 3 & 3 & 4 & 4 & 5 & 29 & 1 \\
\hline $\begin{array}{l}\text { Yad } \\
\text { hipa } \\
\text { orey }\end{array}$ & 1 & 4 & 4 & 6 & 2 & 2 & 2 & 2 & 6 & 29 & 1 \\
\hline $\begin{array}{l}\text { Ore } \\
\text { y } \\
\text { bro } \\
\text { kchi } \\
\text { lu }\end{array}$ & 3 & 6 & 2 & 5 & 6 & 4 & 6 & 5 & 4 & 41 & 6 \\
\hline $\begin{array}{l}\text { Ore } \\
\text { y } \\
\text { regt } \\
\text { ang }\end{array}$ & 6 & 5 & 1 & 1 & 5 & 6 & 3 & 1 & 1 & 29 & 1 \\
\hline $\begin{array}{l}\text { Ker } \\
\text { ong } \\
\text { ree } \\
\text { orey }\end{array}$ & 5 & 2 & 6 & 4 & 1 & 5 & 1 & 3 & 2 & 29 & 1 \\
\hline
\end{tabular}




\begin{tabular}{|l|l|l|l|l|l|l|l|l|l|l|l|}
\begin{tabular}{|l|l|l|l|l|l|l|} 
Bro \\
kpal
\end{tabular} & & & & & & & & & & & \\
$\mathrm{i}$ & 4 & 3 & 3 & 3 & 4 & 1 & 5 & 6 & 3 & 32 & 5 \\
\hline
\end{tabular}

Table 8: Data used for genereation graphs

Leaf area $(\mathbf{d m} 2)$

\begin{tabular}{|l|c|c|}
\hline Genotypes & $\begin{array}{l}\text { Non } \\
\text { stress }\end{array}$ & $\begin{array}{l}\text { Drought } \\
\text { stress }\end{array}$ \\
\hline Orey serbu & 0.75 & 0.55 \\
\hline Yadhipa orey & 0.5 & 0.34 \\
\hline Orey brokchilu & 0.45 & 0.34 \\
\hline Orey regtang & 0.46 & 0.38 \\
\hline Keronngree orey & 0.52 & 0.4 \\
\hline Brokpali & 0.4 & 0.3 \\
\hline Mean & $\mathbf{0 . 5 1}$ & $\mathbf{0 . 4 3}$ \\
\hline SD & $\mathbf{0 . 1 2}$ & $\mathbf{0 . 0 9}$ \\
\hline CV\% & $\mathbf{2 4 . 1 6}$ & $\mathbf{2 0 . 4 7}$ \\
\hline SE & $\mathbf{0 . 0 5}$ & $\mathbf{0 . 0 4}$ \\
\hline
\end{tabular}

Pod length $(\mathrm{cm})$

\begin{tabular}{|l|c|c|}
\hline Genotypes & $\begin{array}{l}\text { Non } \\
\text { stress }\end{array}$ & $\begin{array}{l}\text { Drought } \\
\text { stress }\end{array}$ \\
\hline Orey serbu & 12.42 & 10.68 \\
\hline Yadhipa orey & 8.24 & 7.33 \\
\hline Orey brokchilu & 12.41 & 10.02 \\
\hline Orey regtang & 12.5 & 10.93 \\
\hline Keronngree orey & 13.28 & 12.07 \\
\hline Brokpali & 11.8 & 9.65 \\
\hline Mean & $\mathbf{1 1 . 7 8}$ & $\mathbf{1 0 . 1 1}$ \\
\hline SD & $\mathbf{1 . 7 9}$ & $\mathbf{1 . 6 0}$ \\
\hline CV\% & $\mathbf{1 5 . 2 4}$ & $\mathbf{1 5 . 8 1}$ \\
\hline SE & $\mathbf{0 . 7 3}$ & $\mathbf{0 . 6 5}$ \\
\hline
\end{tabular}


Pod number

\begin{tabular}{|l|c|c|}
\hline Genotypes & $\begin{array}{l}\text { Non } \\
\text { stress }\end{array}$ & $\begin{array}{l}\text { Drought } \\
\text { stress }\end{array}$ \\
\hline Orey serbu & 3.99 & 3 \\
\hline Yadhipa orey & 5.66 & 5.25 \\
\hline Orey brokchilu & 5.83 & 3.33 \\
\hline Orey regtang & 7.39 & 3.94 \\
\hline Keronngree orey & 6.33 & 3.5 \\
\hline Brokpali & 5.05 & 4.94 \\
\hline Mean & $\mathbf{5 . 7 1}$ & $\mathbf{3 . 9 9}$ \\
\hline SD & $\mathbf{1 . 1 5}$ & $\mathbf{0 . 9 1}$ \\
\hline CV\% & $\mathbf{2 0 . 1 5}$ & $\mathbf{2 2 . 8 3}$ \\
\hline SE & $\mathbf{0 . 4 7}$ & $\mathbf{0 . 3 7}$ \\
\hline
\end{tabular}

Root length (cm)

\begin{tabular}{|l|c|c|}
\hline Genotypes & $\begin{array}{l}\text { Non } \\
\text { stress }\end{array}$ & $\begin{array}{l}\text { Drought } \\
\text { stress }\end{array}$ \\
\hline Orey serbu & 28.9 & 24.05 \\
\hline Yadhipa orey & 31.07 & 24.77 \\
\hline Orey brokchilu & 19.77 & 18.38 \\
\hline Orey regtang & 18.24 & 23.5 \\
\hline Keronngree orey & 21.78 & 24.33 \\
\hline Brokpali & 22.83 & 23.44 \\
\hline Mean & $\mathbf{2 3 . 7 7}$ & $\mathbf{2 3 . 0 8}$ \\
\hline SD & $\mathbf{5 . 1 2}$ & $\mathbf{2 . 3 6}$ \\
\hline CV\% & $\mathbf{2 1 . 5 4}$ & $\mathbf{1 0 . 2 1}$ \\
\hline SE & $\mathbf{2 . 0 9}$ & $\mathbf{0 . 9 6}$ \\
\hline
\end{tabular}

Root weight (g)

\begin{tabular}{|l|l|l|}
\hline Genotypes & $\begin{array}{l}\text { Non } \\
\text { stress }\end{array}$ & $\begin{array}{l}\text { Drought } \\
\text { stress }\end{array}$ \\
\hline
\end{tabular}




\begin{tabular}{|l|c|c|} 
Orey serbu & 2.29 & 1.22 \\
\hline Yadhipa orey & 2.55 & 1.60 \\
\hline Orey brokchilu & 1.96 & 1.53 \\
\hline Orey regtang & 2.02 & 1.62 \\
\hline Keronngree orey & 2.9 & 1.44 \\
\hline Brokpali & 2.7 & 1.84 \\
\hline Mean & $\mathbf{2 . 4 0}$ & $\mathbf{1 . 5 4}$ \\
\hline SD & $\mathbf{0 . 3 8}$ & $\mathbf{0 . 2 1}$ \\
\hline CV\% & $\mathbf{1 5 . 7 3}$ & $\mathbf{1 3 . 3 9}$ \\
\hline SE & $\mathbf{0 . 1 5}$ & $\mathbf{0 . 0 8}$ \\
\hline
\end{tabular}

RWC (\%)

\begin{tabular}{|l|c|c|}
\hline Genotypes & $\begin{array}{l}\text { Non } \\
\text { stress }\end{array}$ & $\begin{array}{l}\text { Drought } \\
\text { stress }\end{array}$ \\
\hline Orey serbu & 45.86 & 45.17 \\
\hline Yadhipa orey & 53.09 & 38.32 \\
\hline Orey brokchilu & 60.66 & 48.25 \\
\hline Orey regtang & 46.63 & 46.32 \\
\hline Keronngree orey & 56.4 & 47.69 \\
\hline Brokpali & 63.24 & 57.59 \\
\hline Mean & $\mathbf{5 4 . 3 1}$ & $\mathbf{4 7 . 2 2}$ \\
\hline SD & $\mathbf{7 . 1 6}$ & $\mathbf{6 . 2 1}$ \\
\hline CV\% & $\mathbf{1 3 . 1 8}$ & $\mathbf{1 3 . 1 6}$ \\
\hline SE & $\mathbf{2 . 9 2}$ & $\mathbf{2 . 5 4}$ \\
\hline
\end{tabular}

Shoot weight $(\mathrm{g})$

\begin{tabular}{|l|c|c|}
\hline Genotypes & $\begin{array}{l}\text { Non } \\
\text { stress }\end{array}$ & $\begin{array}{l}\text { Drought } \\
\text { stress }\end{array}$ \\
\hline Orey serbu & 12.73 & 6.91 \\
\hline Yadhipa orey & 8.92 & 3.50 \\
\hline Orey brokchilu & 14.27 & 2 \\
\hline Orey regtang & 13.91 & 5.25 \\
\hline Keronngree orey & 5.67 & 4.75 \\
\hline
\end{tabular}




\begin{tabular}{|l|c|c|} 
Brokpali & 14.61 & 5.94 \\
\hline Mean & $\mathbf{1 1 . 6 8}$ & $\mathbf{4 . 7 1}$ \\
\hline SD & $\mathbf{3 . 6 1}$ & $\mathbf{1 . 7 6}$ \\
\hline CV\% & $\mathbf{3 0 . 8 9}$ & $\mathbf{3 7 . 3 2}$ \\
\hline SE & $\mathbf{1 . 4 7}$ & $\mathbf{0 . 7 2}$ \\
\hline
\end{tabular}

Seed number

\begin{tabular}{|l|c|c|}
\hline Genotypes & $\begin{array}{l}\text { Non } \\
\text { stress }\end{array}$ & $\begin{array}{l}\text { Drought } \\
\text { stress }\end{array}$ \\
\hline Orey serbu & 4.01 & 3.01 \\
\hline Yadhipa orey & 1.83 & 1.89 \\
\hline Orey brokchilu & 3.45 & 2.47 \\
\hline Orey regtang & 4.4 & 4.74 \\
\hline Keronngree orey & 3.28 & 3.36 \\
\hline Brokpali & 3.93 & 2.72 \\
\hline Mean & $\mathbf{3 . 4 8}$ & $\mathbf{3 . 0 3}$ \\
\hline SD & $\mathbf{0 . 9 0}$ & $\mathbf{0 . 9 7}$ \\
\hline CV\% & $\mathbf{2 6 . 0 0}$ & $\mathbf{3 2 . 1 4}$ \\
\hline SE & $\mathbf{0 . 3 7}$ & $\mathbf{0 . 4 0}$ \\
\hline
\end{tabular}

Seed weight (g)

\begin{tabular}{|l|c|c|}
\hline Genotypes & $\begin{array}{l}\text { Non } \\
\text { stress }\end{array}$ & $\begin{array}{l}\text { Drought } \\
\text { stress }\end{array}$ \\
\hline Orey serbu & 12.73 & 5.77 \\
\hline Yadhipa orey & 8.92 & 4.33 \\
\hline Orey brokchilu & 14.27 & 2 \\
\hline Orey regtang & 13.91 & 4.66 \\
\hline Keronngree orey & 5.67 & 4.33 \\
\hline Brokpali & 14.61 & 5.94 \\
\hline Mean & $\mathbf{1 1 . 6 8}$ & $\mathbf{4 . 5 0}$ \\
\hline SD & $\mathbf{3 . 6 1}$ & $\mathbf{1 . 4 2}$ \\
\hline CV\% & $\mathbf{3 0 . 8 9}$ & $\mathbf{3 1 . 4 6}$ \\
\hline
\end{tabular}




\begin{tabular}{|l|l|l|} 
SE & 1.47 & 0.58 \\
\hline
\end{tabular}

Maturity
\begin{tabular}{|l|c|c|}
\hline Genotypes & $\begin{array}{l}\text { Non } \\
\text { stress }\end{array}$ & $\begin{array}{l}\text { Drought } \\
\text { stress }\end{array}$ \\
\hline Orey serbu & 50 & 60 \\
\hline Yadhipa orey & 57 & 66.00 \\
\hline Orey brokchilu & 58.6 & 59.6 \\
\hline Orey regtang & 59 & 65 \\
\hline Keronngree orey & 55.6 & 62 \\
\hline Brokpali & 59.6 & 55.6 \\
\hline Mean & $\mathbf{5 6 . 6 0}$ & $\mathbf{6 1 . 5 0}$ \\
\hline SD & $\mathbf{3 . 5 6}$ & $\mathbf{4 . 2 2}$ \\
\hline CV\% & $\mathbf{6 . 2 9}$ & $\mathbf{6 . 8 5}$ \\
\hline SE & $\mathbf{1 . 4 5}$ & $\mathbf{1 . 8 9}$ \\
\hline
\end{tabular}

